\title{
Automated Construction of Quantum-Classical Hybrid Models
}

\section{Journal Article}

Author(s):

Brunken, Christoph; Reiher, Markus (D)

Publication date:

2021-06-08

Permanent link:

https://doi.org/10.3929/ethz-b-000492817

\section{Rights / license:}

Creative Commons Attribution-NonCommercial-NoDerivatives 4.0 International

\section{Originally published in:}

Journal of Chemical Theory and Computation 17(6), https://doi.org/10.1021/acs.jctc.1c00178

\section{Funding acknowledgement:}

182400 - Exhaustive First-Principles Exploration of Chemical Reaction Networks for Catalysis Design (SNF) 


\title{
Automated Construction of Quantum-Classical Hybrid Models
}

\author{
Christoph Brunken and Markus Reiher*
}

Cite This: J. Chem. Theory Comput. 2021, 17, 3797-3813

Read Online

ABSTRACT: We present a protocol for the fully automated construction of quantum mechanical (QM)-classical hybrid models by extending our previously reported approach on selfparametrizing system-focused atomistic models (SFAMs) [Brunken, C.; Reiher, M. J. Chem. Theory Comput. 2020, 16, 3, 1646-1665]. In this QM/SFAM approach, the size and composition of the QM region are evaluated in an automated manner based on first principles so that the hybrid model describes the atomic forces in the center of the QM region accurately. This entails the automated construction and evaluation of differently sized QM regions with a bearable computational overhead that needs to be paid for automated validation procedures. Applying SFAM for the classical part of the model eliminates any dependence on pre-existing parameters due to its system-focused quantum mechanically derived parametrization. Hence, QM/SFAM is capable of delivering high fidelity and complete automation. Furthermore, since SFAM parameters are generated for the whole system, our ansatz allows for a convenient redefinition of the QM region during a molecular exploration. For this purpose, a local reparametrization scheme is introduced, which efficiently generates additional classical parameters on the fly when new covalent bonds are formed (or broken) and moved to the classical region.

\section{INTRODUCTION}

In contrast to most protocols of computational quantum chemistry that consider isolated molecules, chemical processes can take place in a vast variety of complex environments. Studying chemical reactions in proteins, in nanostructures, and on surfaces requires a theoretical approach that must provide an accurate quantum mechanical description of the reaction center and at the same time an efficient model to cope with the enormous system size of the structured, heterogeneous environment. For these requirements to coalesce, one is forced to apply a hybrid method, which divides the system into several regions treated at different levels of approximation. ${ }^{1,2}$

Typically, the reaction center is modeled with a quantum mechanical method, which allows one to describe the formation and cleavage of covalent bonds in a natural way. The environment may efficiently be treated by a force field ${ }^{3}$ rooted in classical mechanics. Such a quantum-mechanical/ molecular-mechanical hybrid (QM/MM) model ${ }^{4,5}$ typically requires significant manual work involved in the model construction process. It is plagued by a lack of standardization and comparability, and rigorous uncertainty quantification is not available (as for most computational chemistry methods), which is particularly critical for such complex composite models. Typically, no standardized procedures exist regarding, for instance, the parametrization of the force field (especially for metal-containing regions), the QM region determination, the choice of boundary scheme, the initial structure generation, conformational sampling, and the extent to which parts of the macromolecule are constrained during structure optimizations to confine the complexity of the system, as well as to limit the error of the MM energy contribution.

One of the most prominent challenges for QM/MM modeling is the construction of an efficient and at the same time accurate molecular mechanics model, which is typically only available for a predefined subset of chemical elements with standard bonding patterns. Its degree of transferability to a new system is not obvious at all. We addressed this issue in our recent work ${ }^{6}$ by introducing the self-parametrizing systemfocused atomistic model (SFAM), which allows for automated construction of a molecular mechanics model for a system of arbitrary size and elemental composition. The reference data for the model are obtained in a fully automated way by an autonomous fragmentation algorithm and subsequent quantum chemical reference calculations for the molecular fragments. Furthermore, SFAM includes a model refinement step based on $\Delta$-machine learning $(\mathrm{ML})^{7}$ when additional reference data become available during a molecular exploration.

In this work, we extend SFAM toward quantum-classical hybrid models (QM/SFAM) with an automated setup. The

Received: February 18, 2021

Published: May 12, 2021 
application of SFAM as the classical part of the model eliminates any issues arising from an incomplete set of parameters. Furthermore, SFAM guarantees that the reference data, from which the MM parameters are derived, can be provided by the same quantum chemical method as is applied in the QM part of the hybrid calculation making the MM model as consistent as possible with the QM part.

Furthermore, we present a scheme to determine the choice of the QM region, which results in an accurate QM/SFAM model. The selection of atoms to include in the quantum mechanical part of the calculation is a highly nontrivial task and the decision often relies on chemical intuition alone. However, there have been recent efforts to select QM regions systematically on a first-principles basis by Kulik and coworkers. ${ }^{8}$ Their systematic QM region determination scheme relies on evaluating how specific residues of a protein affect the electronic structure (charge distribution, frontier orbitals) of the core residues of the protein. In this work, we provide an alternative approach solely based on the fundamental quantities of a molecular system, i.e., its electronic energy and its derivatives with respect to the nuclear coordinates, which are available from computationally cheap and approximate, as well as expensive and accurate, electronic structure models.

The nuclear derivatives play an essential role in obtaining reliable structures (by molecular dynamics (MD) sampling or structure optimization) and we therefore focus on the accurate description of the atomic forces when determining which atoms must be included in the quantum mechanical part of the model. Defining an optimal set of atoms as the QM region is essential, especially because it has been demonstrated by Ochsenfeld, ${ }^{9-11}$ Martinez, ${ }^{12}$ and others ${ }^{13-15}$ that for many systems QM/MM models safely converge only with large QM region sizes of several hundred atoms. Therefore, in those cases where such large QM regions are not feasible (e.g., vast reaction network explorations or MD simulations), it is inevitable to carefully select the QM region systematically to guarantee that the resulting model is an accurate approximation to a full quantum mechanical model.

In the following, we first describe our QM/SFAM model and then introduce an algorithm to systematically determine the composition of the QM region in an automated way. This is demonstrated with the examples of (i) a medium-sized peptide that also allows for full-quantum reference calculations and (ii) a larger system to resemble a typical case of application. Although these examples are taken from biochemistry, we emphasize that our model is agnostic with respect to the elemental composition due to its first-principles core. Hence, any nanoscale atomistic system can be subjected to our hybrid model construction process, even one for which a molecular structure first needs to be constructed (by virtue of the SFAM approach that early on in the model generation provides an approximate force field for iterative structure refinement ${ }^{6}$ ).

\section{THEORY}

2.1. SFAM Approach. We briefly review the SFAM approach $^{6}$ as it is the classical part of our hybrid model. Similar to $\mathrm{QMDFF}^{16}$ and QuickFF ${ }^{17}$ molecular mechanics models, SFAM is also generated automatically for a specific molecular system from quantum mechanical reference data, which yields accurate force fields without being limited by the elemental composition of the molecular system. SFAM is distinct from the two aforementioned models ${ }^{16,17}$ in two crucial aspects. First, SFAM force constants are parametrized by a partial Hessian fit algorithm ${ }^{18,19}$ as introduced by Hirao and coworkers in 2016, i.e., the parameters are fitted solely to local information in the Hessian, which allows us to generate the model for very large molecular systems by calculating reference data for fragments cut out of the whole structure. We also introduced an autonomous fragmentation algorithm for this purpose. ${ }^{6}$ Second, SFAM includes an (optional) improvement step based on $\Delta$-machine learning. ${ }^{7}$

While the MM base model of SFAM provides an accurate description of the potential energy surface (PES) close to the local energy minimum taken as a reference for parametrization, its parameters are not guaranteed to be transferable across all regions of the PES. The base model can be applied in an exploration of additional structures (e.g., in molecular dynamics simulations), for which additional reference data can be calculated on the fly. The MM/ML ansatz of SFAM can then gradually increase its accuracy across the PES as an increasing amount of reference data is collected to train the ML model. Many ML-only models have been reported as replacements for classical force fields. ${ }^{20-24}$ However, our MM/ $\mathrm{ML}$ approach for SFAM has several advantages. On the one hand, the MM base model provides physical insight into the properties of the system in contrast to an approach solely based on ML. On the other hand, it requires only a limited and well controllable amount of reference data, as it is parametrized on single-point data obtained for fragments (optimized structures, atomic charges, and Hessians).

The SFAM energy $E_{\text {SFAM }}$ can be written as the sum of the $\mathrm{MM}$ and $\mathrm{ML}$ contributions

$$
E_{\mathrm{SFAM}}=E_{\mathrm{MM}}+E_{\mathrm{ML}}
$$

$E_{\mathrm{ML}}$ is zero at this level because we choose the hybrid QM/ SFAM model as the new base model, which can then be refined in a later step by $\Delta-\mathrm{ML}$. The SFAM energy expression is divided into a covalent (cov) part $E_{\text {cov }}$ and nonbonding (nb) potential energy contributions $E_{\mathrm{nb}}$

$$
E_{\mathrm{SFAM}}=E_{\mathrm{cov}}+E_{\mathrm{nb}}
$$

as is common in MM models. ${ }^{3}$ The covalent energy contribution is calculated from the displacements of the internal degrees of freedom out of their equilibrium positions and can therefore be divided into terms for bonds $r$, bond angles $\alpha$, dihedral angles $\theta$, and improper dihedral angles $\varphi$

$$
\begin{aligned}
E_{\mathrm{cov}} & =E_{r}+E_{\alpha}+E_{\theta}+E_{\varphi} \\
& =\sum_{(A, B)} E_{r}^{A B}+\sum_{(A, B, C)} E_{\alpha}^{A B C}+\sum_{(A, B, C, D)} E_{\theta}^{A B C D} \\
& +\sum_{(X, B, C, D)} E_{\varphi}^{X B C D}
\end{aligned}
$$

where $(A, B)$ denotes a group of two bonded atoms $A$ and $B$, $(A, B, C)$ a bonded triplet of atoms, $(A, B, C, D)$ a bonded quadruplet of atoms, and $(X, B, C, D)$ the atoms of an improper dihedral angle (with $X$ representing the center atom). The nonbonding interactions comprise an electrostatic part (estat), dispersive (disp) and Pauli repulsion (rep) interactions, and hydrogen bonds 


$$
\begin{aligned}
E_{\mathrm{nb}} & =E_{\text {estat }}+E_{\text {disp }}+E_{\text {rep }}+E_{\mathrm{hb}} \\
& =\sum_{(A, B)}\left(E_{\text {estat }}^{A B}+E_{\text {disp }}^{A B}+E_{\text {rep }}^{A B}\right)+\sum_{(D, H, A)} E_{\mathrm{hb}}^{D H A}
\end{aligned}
$$

where $(A, B)$ represents a pair of atoms, in which the atoms $A$ and $B$ are neither bonded to one another nor both bonded to another atom $C$, and $(D, H, A)$ is a hydrogen bond. For details on the potential energy expressions for each of the MM contributions, as well as an explanation of the parametrization procedure, we refer to our previous paper. ${ }^{6}$

We emphasize that combining the classical model in SFAM with a quantum chemical method creates an opportunity to apply our $\Delta$-ML improvement step to the hybrid model. During a molecular exploration with the QM/SFAM method, quantum chemical reference data are collected without any additional effort. Our machine-learned model corrections can be trained with these data and are valuable (i) if the QM focus is moved to a different section of the whole system and (ii) if the data can be transferred to improve the description of similar molecular substructures located in the MM region. We also note that related efforts to combine machine learning with quantum-classical hybrid methods have been reported recently. ${ }^{25,26}$

2.2. Hybrid QM/SFAM Formalism. The energy expression of any hybrid model with two distinct regions, a quantum core $Q$ and an environment $\mathcal{E}$, can be approximately separated into a quantum-core-only contribution $E^{Q}$, an analogous contribution of the environment $E^{\mathcal{E}}$, and an interaction energy $E^{Q-\mathcal{E}}$

$$
E_{\text {hybrid }}=E^{Q}+E^{\mathcal{E}}+E^{Q-\mathcal{E}}
$$

When choosing two different methods for the two regions $Q$ and $\mathcal{E}$, there must not be any electromagnetic interaction included twice or be missing completely. As noted before, we apply a quantum chemical method for the core region and SFAM for the environment. Furthermore, we distinguish two schemes for the interaction energy $E^{Q-\mathcal{E}}$ : (a) one in which the electrostatic interaction is treated by SFAM and (b) one in which it is described quantum mechanically. The former is known as mechanical embedding (ME) and the latter as electrostatic embedding (EE). Although it has been demonstrated that EE provides more accurate results than $\mathrm{ME}$, in particular for small QM regions, ${ }^{11}$ we implemented both embedding schemes because EE may not always be available for the QM method of choice. In the case of ME, the QM/ SFAM energy expression reads as follows

$$
\begin{aligned}
E_{\mathrm{QM} / \mathrm{SFAM}}^{\mathrm{ME}} & =E_{\mathrm{SFAM}}^{Q+\mathcal{E}}+E_{\mathrm{QM}}^{Q}-\sum_{\substack{(A, B) \\
\in Q}} E_{r}^{A B}-\sum_{\substack{(A, B, C) \\
\in Q}} E_{\alpha}^{A B C} \\
& -\sum_{\substack{(A, B, C, D) \\
\in Q}} E_{\theta}^{A B C D}-\sum_{\substack{(X, B, C, D) \\
\in Q}} E_{\varphi}^{X B C D} \\
& -\sum_{\substack{A \in Q \\
B \in Q}}\left(E_{\mathrm{estat}}^{A B}+E_{\mathrm{disp}}^{A B}+E_{\mathrm{rep}}^{A B}\right)-\sum_{\substack{(D, H, A) \\
\in Q}} E_{\mathrm{hb}}^{D H A}
\end{aligned}
$$

In eq 6 , the energy $E_{\text {SFAM }}^{Q+\mathcal{E}}$ refers to the SFAM energy of the full system (i.e., $Q$ and $\mathcal{E}$ combined) and $E_{\mathrm{QM}}^{Q}$ is the electronic energy obtained in a $Q M$ calculation for $Q$,

$$
E_{\mathrm{QM}}^{Q}=\left\langle\hat{H}_{\mathrm{el}, \mathrm{ME}}^{Q}\right\rangle
$$

with the electronic Hamiltonian in atomic units

$$
\begin{aligned}
\hat{H}_{\mathrm{el}, \mathrm{ME}}^{Q}= & -\sum_{i=1}^{N_{\mathrm{el}}^{Q}} \frac{1}{2 m_{e}} \Delta_{i}-\sum_{\alpha=1}^{N_{\mathrm{nuc}}^{Q}} \frac{1}{2 M_{\alpha}} \Delta_{\alpha}-\sum_{i=1}^{N_{\mathrm{el}}^{Q}} \sum_{\alpha=1}^{N_{\mathrm{nuc}}^{Q}} \frac{Z_{\alpha}}{\left|\mathbf{r}_{i}-\mathbf{R}_{\alpha}\right|} \\
& +\sum_{i=1}^{N_{\mathrm{el}}^{Q}} \sum_{j=i+1}^{N_{\mathrm{el}}^{Q}} \frac{1}{\left|\mathbf{r}_{i}-\mathbf{r}_{j}\right|}+\sum_{\alpha=1}^{N_{\mathrm{nuc}}^{Q}} \sum_{\beta=\alpha+1}^{N_{\mathrm{nuc}}^{Q}} \frac{Z_{\alpha} Z_{\beta}}{\left|\mathbf{R}_{\alpha}-\mathbf{R}_{\beta}\right|}
\end{aligned}
$$

where $N_{\mathrm{el}}^{Q}$ is the number of electrons, $N_{\text {nuc }}^{Q}$ is the number of atomic nuclei, $Z_{\alpha}$ is the nuclear charge of nucleus $\alpha$, and $\mathbf{r}_{i}$ and $\mathbf{R}_{\alpha}$ are the Cartesian coordinates of electrons and nuclei in $Q$, respectively.

The latter part of eq 6 subtracts all energy contributions in the $\mathrm{MM}$ force field that are covered by $E_{\mathrm{QM}}^{Q}$, i.e., all bond terms $E_{r}^{A B}$ with the atoms $A$ and $B$ both in the $Q M$ region, all angle terms with at least two, dihedral terms with at least three, and all improper dihedral terms with all four of their corresponding atoms in the QM region. All pairwise noncovalent interaction terms are subtracted for each pair of atoms in $Q$. Hence, all noncovalent interactions between $Q$ and $\mathcal{E}$ are described by SFAM, which is a consistent approach if the electronic structure model does not account for dispersive interactions (as in many standard density functionals) so that they can be treated semiclassically everywhere in the system. For EE, we apply a quantum mechanical description of the electrostatic interaction by defining $E_{\mathrm{QM} / \mathrm{SFAM}}^{\mathrm{EE}}$ as

$$
E_{\mathrm{QM} / \mathrm{SFAM}}^{\mathrm{EE}}=E_{\mathrm{QM} / \mathrm{SFAM}}^{\mathrm{ME}}+E_{\mathrm{QM}, \text { estat }}^{Q}-\sum_{\substack{A \in Q \\ B \in \mathcal{E}}} E_{\text {estat }}^{A B}
$$

with

$$
E_{\mathrm{QM}, \mathrm{estat}}^{Q}=E_{\mathrm{QM}, \mathrm{EE}}^{Q}-E_{\mathrm{QM}, \mathrm{ME}}^{Q}
$$

Eq 9 includes an additional energy contribution obtained in

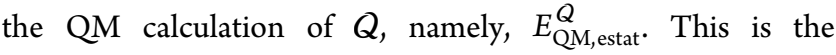
interaction energy of the elementary particles (electrons and nuclei) in $Q$ with the electrostatic potential generated by SFAM's atomic partial charges located at atomic positions in $\mathcal{E}$ as point charges. Naturally, the classical equivalent of this interaction must be subtracted to avoid double counting. The electronic Hamiltonian operator (in Hartree atomic units) is therefore different for $\mathrm{EE}$ compared to $\mathrm{ME}$

$$
\hat{H}_{\mathrm{el}, \mathrm{EE}}^{Q}=\hat{H}_{\mathrm{el}, \mathrm{ME}}^{Q}-\sum_{i=1}^{N_{\mathrm{el}}^{Q}} \sum_{A \in \mathcal{E}} \frac{q_{A}}{\left|\mathbf{r}_{i}-\mathbf{r}_{A}\right|}+\sum_{\alpha=1}^{N_{\mathrm{nuc}}^{Q}} \sum_{A \in \mathcal{E}} \frac{Z_{\alpha} q_{A}}{\left|\mathbf{R}_{\alpha}-\mathbf{r}_{A}\right|}
$$

Here, $q_{A}$ is the partial charge of atom $A$ in $\mathcal{E}$; as before, $Z_{\alpha}$ is the nuclear charge of nucleus $\alpha$ in $Q$; and $\mathbf{r}_{A}, \mathbf{r}_{i}$, and $\mathbf{R}_{\alpha}$ are the Cartesian coordinates of the atoms in $\mathcal{E}$ and the electrons and nuclei in $Q$, respectively. The van der Waals interactions are treated at the SFAM level (based on semiclassical dispersion corrections $^{27-30}$ of Grimme) in both embedding schemes. 
Within $Q$, the $Q M$ method must take care of dispersive interactions.

2.3. QM-SFAM Boundary. The challenge of describing a single molecular system with two different physical theories becomes most apparent at the boundary of the two regions $Q$ and $\mathcal{E}$, particularly if the boundary intersects a covalent chemical bond. ${ }^{31}$ Various strategies have been developed for modeling this QM-MM boundary. ${ }^{1,2}$ By far, the most common one is the link-atom approach,,$^{31-37}$ in which the covalent bond at the border of the QM region is valencesaturated by a hydrogen atom or some other prototypical residue (e.g., a methyl group). The most prominent alternative is to generate localized bond orbitals from a slightly larger QM calculation and include these doubly occupied orbitals in the QM calculation of the hybrid method. During the selfconsistent field (SCF) optimization of the orbitals, these artificial orbitals are kept frozen. This approach known as the local-SCF method ${ }^{38-41}$ was introduced by Rivail and coworkers and later extended by Gao and co-workers. ${ }^{42-44}$ Furthermore, advanced embedding approaches may be applied to separate the QM region from an environment, such as projector-based embedding and embedded mean-field theory, ${ }^{45-49}$ frozen density embedding, ${ }^{50-61}$ or the subsystem separation by unitary block-diagonalization approach (SSUB). ${ }^{62}$

In our QM/SFAM implementation, we focus on the linkatom approach but emphasize that our implementation can be extended to include the more advanced boundary schemes mentioned above. It is crucial for the link-atom approach to carefully select the bonds that indicate the boundary of the $\mathrm{QM}$ region because replacing heavy atoms with hydrogen link atoms introduces artificial effects on neighboring molecular entities by distorting the electronic structure.

Our automated strategy for placing link atoms is described in Section 2.7. Along the vector of a single bond $i$

$$
\mathbf{r}_{\mathcal{E}_{i} Q_{i}}=\mathbf{r}_{\mathcal{E}_{i}}-\mathbf{r}_{Q_{i}}
$$

which is defined by the coordinates of atom $\mathcal{E}_{i}$ in the environment and $Q_{i}$ of the quantum region bonded to $\mathcal{E}_{i}$, a hydrogen link atom is positioned at

$$
\mathbf{r}_{\mathrm{H}_{i}}=\mathbf{r}_{Q_{i}}+\left(R_{Q_{i}, \mathrm{cov}}+R_{\mathrm{H}, \mathrm{cov}}\right) \frac{\mathbf{r}_{\mathcal{E}_{i} Q_{i}}}{\left|\mathbf{r}_{\mathcal{E}_{i} Q_{i}}\right|}
$$

with the covalent radii ${ }^{63,64} R_{Q_{i} \text {,cov }}$ and $R_{H, \text { cov }}$ of $Q_{i}$ and hydrogen. This approach allows us to cut through single bonds only (see Section 2.7), which is, however, not a severe restriction, especially considering the fact that the QM region can be enlarged to eventually meet it.

To exploit the force on an artificial link atom $\mathrm{H}_{i}$, which is located between $\mathcal{E}_{i}$ and $Q_{i}$, its energy gradient $\mathbf{g}_{\mathrm{H}_{i}}$ calculated in the QM calculation must be distributed to $\mathcal{E}_{i}$ and $Q_{i}$. The gradient contributions $\tilde{\mathbf{g}}_{\mathcal{E}_{i}}$ and $\tilde{\mathbf{g}}_{Q_{i}}$ in direction $\mu(\mu=x, y, z)$ are, following ref 65

$$
\begin{aligned}
& \tilde{g}_{Q_{i}, \mu}=\mathbf{g}_{\mathrm{H}_{i}}^{\mathrm{T}} \cdot\left(\left(1-\frac{\left|\mathbf{r}_{Q_{i}}-\mathbf{r}_{\mathrm{H}_{i}}\right|}{\left|\mathbf{r}_{\mathcal{E}_{i} Q_{i}}\right|}\right) \mathbf{u}_{\mu}+\frac{\left|\mathbf{r}_{Q_{i}}-\mathbf{r}_{\mathrm{H}_{i}}\right| d_{\mu}}{\mid \mathbf{r}_{\left.\mathcal{E}_{i} Q_{i}\right|^{3}}} \mathbf{r}_{\mathcal{E}_{i} Q_{i}}\right) \\
& \tilde{g}_{\mathcal{E}_{i}, \mu}=\mathbf{g}_{\mathrm{H}_{i}}^{\mathrm{T}} \cdot\left(\frac{\left|\mathbf{r}_{Q_{i}}-\mathbf{r}_{\mathrm{H}_{i}}\right|}{\mid \mathbf{r}_{\mathcal{E}_{i} Q_{i} \mid}} \mathbf{u}_{\mu}-\frac{\left|\mathbf{r}_{Q_{i}}-\mathbf{r}_{\mathrm{H}_{i}}\right| d_{\mu}}{\mid \mathbf{r}_{\left.\mathcal{E}_{i} Q_{i}\right|^{3}}} \mathbf{r}_{\mathcal{E}_{i} Q_{i}}\right)
\end{aligned}
$$

where $d_{\mu}$ is the absolute value of the difference in the $\mu$ th component of $\mathbf{r}_{\mathcal{E}_{i}}$ and $\mathbf{r}_{Q_{i}}$, and $\mathbf{u}_{\mu}$ is a unit vector in direction $\mu$, e.g., $\mathbf{u}_{z}=(0,0,1)^{\mathrm{T}}$. With these equations, it is straightforward to calculate analytic gradients for the QM/SFAM energy as long as analytic gradients are available for the QM method (including gradients on the external point charges). These gradients are essential for efficient structure optimizations and for molecular dynamics simulations with our QM/SFAM model.

In electrostatic embedding, another issue arises at the $Q-\mathcal{E}$ boundary. Since the partial charge $q_{\mathcal{E}_{i}}$ of some atom $\mathcal{E}_{i}$ at the boundary is included in the QM calculation as an external point charge, the link atom may suffer from overpolarization effects caused by the close proximity of this charge. To counteract this artificial effect, many strategies have been proposed such as deleting the charge, ${ }^{32,66,67}$ redistributing it, $^{68-72}$ or smearing it out ${ }^{38,73,74}$ by replacing the point charge by a Gaussian charge distribution centered at $\mathcal{E}_{i}$. We apply a charge redistribution scheme that was shown to produce accurate results compared to full QM calculations. ${ }^{72} \mathrm{We}$ implemented two variants of charge redistribution: (i) one in which the total charge is conserved (redistribution of charge, denoted as RC) and (ii) one in which also the bond dipoles of the first shell of bonds in $\mathcal{E}$ are conserved (redistribution of charge and dipoles, denoted as RCD). Both schemes are illustrated in Figure 1. (a)

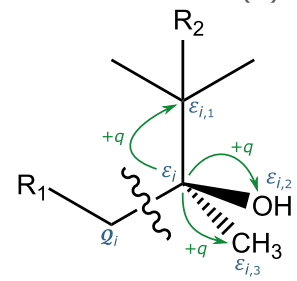

(b)

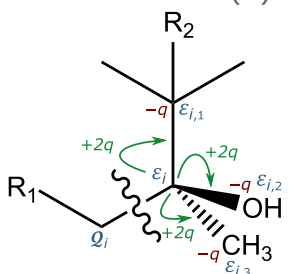

Figure 1. Illustration of (a) the redistribution of charge (RC) scheme and (b) the redistribution of charge and dipoles (RCD) scheme to prevent overpolarization of a link atom (not shown) by the charge on $\mathcal{E}_{i}$ at the QM/SFAM boundary $i$ (wiggly line). Green highlights the redistribution of partial charges toward the positions to which the arrows are pointing, whereas red indicates a subtraction of charge. We define $q=q_{\mathcal{E}_{i}} / n$ with $n$ being the number of non-QM neighbors of $\mathcal{E}_{i}$. In this example, $n=3$ as the neighboring atoms are $\mathcal{E}_{i, 1}, \mathcal{E}_{i, 2}$, and $\mathcal{E}_{i, 3} . R_{1}$ represents the remainder of the $\mathrm{QM}$ region and $R_{2}$ denotes the rest of the environment.

In both schemes, the charge on $\mathcal{E}_{i}$ vanishes; i.e., the new charge is $\tilde{q}_{\mathcal{E}_{i}}=0$. In the RC scheme, the charge is shifted equally to the $n$ neighbors $\mathcal{E}_{i, k}$ (with $k \in\{1, \cdots, n\}$ ) of $\mathcal{E}_{i}$, which are also in $\mathcal{E}$

$$
\tilde{q}_{\mathcal{E}_{i, k}}=\frac{q_{\mathcal{E}_{i}}}{n}, \text { for } k=1, \ldots, n
$$

In the RCD scheme, to conserve the bond dipoles of the bonds $\mathcal{E}_{i}-\mathcal{E}_{i, k}$, the charge on $\mathcal{E}_{i}$ is shifted to the positions half-way in between the $\mathcal{E}_{i}-\mathcal{E}_{i, k}$ bond vectors and doubled in magnitude, resulting in auxiliary charges at positions $\mathbf{r}_{\text {aux }, k}$

$$
\tilde{q}_{\mathbf{r}_{\text {aux }, k}}=\frac{2 q_{\mathcal{E}_{i}}}{n}
$$


with

$$
\mathbf{r}_{\mathrm{aux}, k}=\frac{1}{2}\left(\mathbf{r}_{\mathcal{E}_{i}}+\mathbf{r}_{\mathcal{E}_{i, k}}\right)
$$

A factor of 2 , i.e., the doubling of the shifted charge, is introduced to preserve the magnitude of the bond dipole as the distance between the charges is halved. Consequently, also, the charges on the neighboring atoms must be adjusted so that the new charges on atoms $\mathcal{E}_{i, k}$ become

$$
\tilde{q}_{\mathcal{E}_{i, k}}=q_{\mathcal{E}_{i, k}}-\frac{q_{\mathcal{E}_{i}}}{n}
$$

2.4. QM/SFAM Structure Optimization. As targets of QM/SFAM are large systems with many degrees of freedom, structure optimizations tend to require many iterations to reach convergence. Most of the degrees of freedom to optimize can be attributed to the environment $\mathcal{E}$ and therefore the necessity to perform a $\mathrm{QM}$ calculation in every optimization step can be avoided by a microiteration-based structure optimization. Several variants of such an algorithm exist and have been implemented in QM/MM programs to accelerate structure optimizations of large systems. ${ }^{75-81}$ The aim of these approaches is to reach the same local energy minimum structure as in a regular optimization without expensive QM calculations in every step.

In our variant of the algorithm, the Cartesian coordinates of all atoms in $Q$ and those of atoms in $\mathcal{E}$ within a distance $R$ to any atom in $Q$ are frozen, while all remaining MM degrees of freedom are relaxed (either until convergence or until a maximum of $N_{1}$ steps is reached). As all of the atoms in $Q$ are fixed (i.e., their gradients are treated as zero), no $\mathrm{QM}$ calculation is necessary during these microiterations. We note that despite the system-focused parametrization of SFAM that can be tailored to the QM model in QM/SFAM, our attempts to utilize the MM gradient for the whole system in this step were fruitless. In fact, it is this remaining mismatch of SFAM and $\mathrm{QM}$ forces that requires the environment atoms at the QM boundary to be kept frozen.

Then, the complete system is relaxed according to the full QM/SFAM gradients. No nuclear positions are constrained and therefore a QM calculation is needed for each evaluation of the complete gradient. Once convergence is reached, the optimization terminates. If convergence cannot be reached after $\mathrm{N}_{2}$ steps, then one macroiteration step is completed and the procedure iterates again starting with the first MM-only step.

For the parameters of this algorithm, we found values of $R=$ $4 \AA, N_{1}=1000$, and $N_{2}=15$ to perform well in all examples studied in this work, but they may be adjusted if needed. For the individual structure optimizations, the algorithms implemented in the SCINE Utilities library ${ }^{82}$ are applied.

2.5. QM/SFAM in Molecular Explorations. A crucial issue of molecular mechanics models is that their error in the total energy of the system is expected to scale unfavorably with the system size, e.g., measured in terms of the number of atoms $N_{\text {at }}$. While for small systems, energies obtained with molecular mechanics have been shown to be accurate, especially with system-focused models; ${ }^{6,16,17}$ this is not expected for large systems, which we illustrate using a simple statistical model. ${ }^{83}$ Consider the covalent terms in the total $\mathrm{MM}$ energy expression, which is a sum of approximately $3 N_{\text {at }}$ (mostly) independent terms, each with an uncertainty of $\pm \Delta \varepsilon$. We can model the estimated error under the ideal assumption of equal probabilities to either underestimate or overestimate the energy of a single potential term by $\Delta \varepsilon$. For large $N_{\mathrm{at}}$ the corresponding binomial distribution can be approximated by the normal distribution. ${ }^{84}$ As a result, we estimate a total error of at least

$$
\Delta E \approx 0.675 \sqrt{3 p(1-p) N_{\mathrm{at}}}
$$

to occur with a probability of $50 \%$ on applying these simple assumptions, with $p$ being the probability of overestimating individual potential energy by $\Delta \varepsilon$ instead of underestimating it. For example, for a system with 1000 atoms, it is expected that with a probability of $50 \%$, a total error of at least $18.5 \Delta \varepsilon$ is observed, which scales with $\sqrt{N_{\text {at }}}$. Moreover, the MM model may exhibit a systematic over- or underestimation of the potential energies to some (minor) extent, resulting in an expected error that scales linearly with the system size. Furthermore, we note that the MM noncovalent pair interaction terms, which outnumber the covalent terms, are more difficult to assess with respect to their error contribution ${ }^{85,86}$ because of their distance and hence structure dependence.

Regardless of the simplified assumptions inherent to eq 20, such as neglecting additional uncertainties introduced by the noncovalent interactions (see also refs 85,86 ), it is apparent that for large systems the energies of classical models may exhibit large uncertainties (even with system-focused approaches). By contrast, atomic forces are local quantities evaluated as partial first-order derivatives at a given reference structure for each atomic nucleus.

In view of these considerations, a common practice in QM/ MM studies is to generate and sample structures, either by molecular dynamics simulations or structure optimizations. ${ }^{87-93}$ To obtain accurate energies of local minima on the PES, it is common to freeze all MM atoms beyond a given distance from the active site during structure optimizations ${ }^{90,94-97}$ to obtain a converged structure at smaller computational costs and with a larger resemblance of a reference structure, such as a structure measured by $\mathrm{X}$-ray diffraction, ${ }^{98}$ and to eliminate the contribution of most of the MM region to the total energy. The advantage of this strategy, compared to neglecting all MM contributions to the total energy, is that effects of structural changes close to the active site are captured. However, there exist no standardized guidelines for the choice of this additional cutoff parameter, which may have a significant effect on calculated energies.

Considering all of the aforementioned factors, we introduce a reduced QM/SFAM energy $E_{\mathrm{OM} / \mathrm{SFAM}}^{\mathrm{red}}$ to counteract the possibly large uncertainties induced by the classical description of a large environment $\mathcal{E}$

$$
E_{\mathrm{QM} / \mathrm{SFAM}}^{\mathrm{red}}=E_{\mathrm{QM}}^{Q}+E^{Q-\mathcal{E}}
$$

in which any covalent SFAM contributions and the noncovalent interactions within the environment are neglected. The QM calculation is embedded into the environment by including the $Q-\mathcal{E}$ interaction $E^{Q-\mathcal{E}}$ either through mechanical or electrostatic embedding (see Section 2.2).

We propose that during a molecular exploration, relevant structures should be identified with the complete QM/SFAM model (e.g., by molecular dynamics simulations or structure optimizations), while for the energy differences of intermediate 

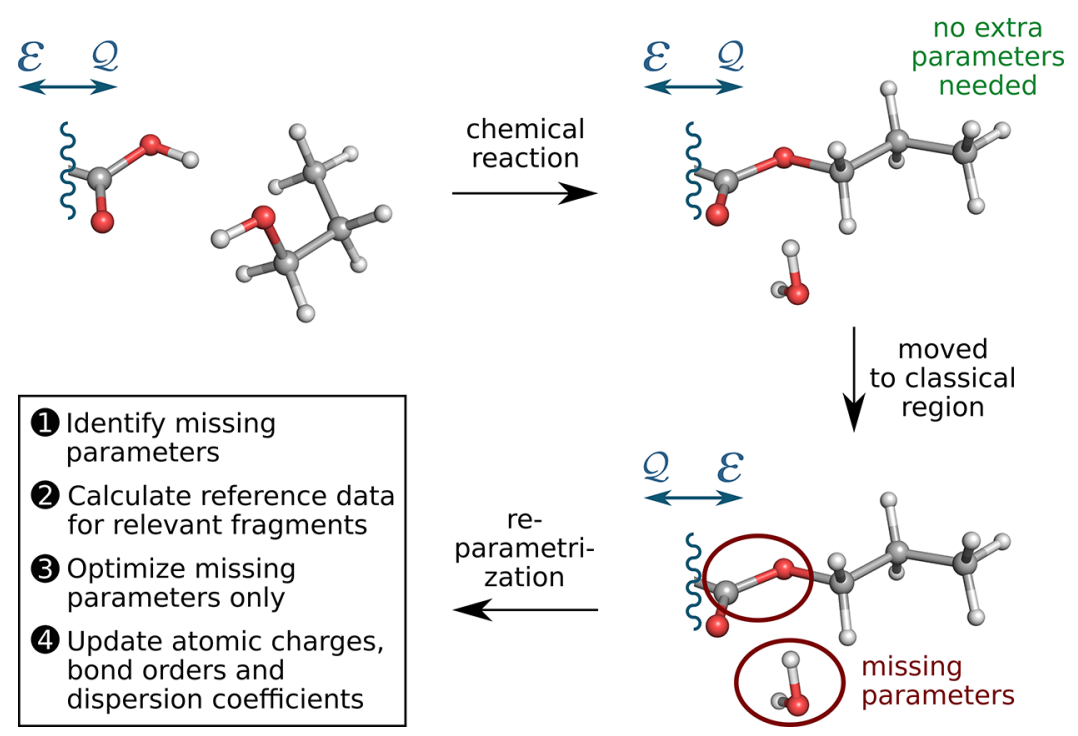

Figure 2. Local reparametrization of the SFAM model if a region in which new covalent bonds are formed or broken is moved out of the QM region $Q$ to become part of the environment $\mathcal{E}$. An esterification reaction, which is embedded in a large environment (not shown for the sake of clarity), is shown. The box provides a short summary of the steps involved in a local model reparametrization. To avoid confusion, we emphasize that each of the three depicted molecular systems are identical with respect to the number and the type of their atoms: the structure on the lefthand side illustrates the reactant and the two structures on the right-hand side show the products of the esterification reaction.

structures on the PES, the difference between the two strategies for computing the energy

$$
\Delta=\Delta E_{\mathrm{QM} / \mathrm{SFAM}}-\Delta E_{\mathrm{QM} / \mathrm{SFAM}}^{\mathrm{red}}
$$

should be monitored closely. For $\Delta E_{\mathrm{QM} / \mathrm{SFAM}}^{\mathrm{red}}$ it is crucial that energy contributions from structural changes in close proximity to the active site are picked up by the QM calculation.

2.6. Automated Reparametrization for Flexible QM Region Definitions. With SFAM as the classical part of the hybrid approach, parameters are always generated for the whole system automatically before starting a molecular exploration. Therefore, one is not restricted in the selection of the QM region and may freely redefine the QM region. It can be valuable to have this flexibility in automated reaction network explorations ${ }^{99,100}$ and in reactive molecular dynamics simulations because reactive centers can shift during a multistep mechanism. This feature is also a requirement for applying QM/SFAM in an interactive quantum chemistry framework, ${ }^{101,102}$ as the ability of the operator to choose a region of interest in a large system should not be limited by missing parameters.

Naturally, QM/SFAM does not require parameters for the covalent terms in the QM region. This means that a reaction that takes place in the QM region and modifies the local connectivity of the atoms (and hence the SFAM topology) does not result in the model becoming unapplicable. Note that parameters for van der Waals interactions, namely, the dispersion coefficients, may be required in the QM region. These can be either quickly re-evaluated or, as an approximation, kept constant even after the modification of the topology because the dispersion coefficients for the same types of elements are expected to be similar (we note that the dispersion coefficients of the predecessor of D3, i.e., D2, ${ }^{103}$ are fixed for each pair of elements). Partial charges are expected to be less transferable after a chemical reaction; however, these are not needed for atoms in the QM region.
Even if no bond breaking and bond formation processes are possible in the classical region, a redefinition of the QM region can move atoms affected by such processes from the QM region to the environment. In this case, the connectivity of the atoms is modified in the classical region and therefore SFAM must be reparametrized if the newly required SFAM parameters are not available due to the existence of the same bonding pattern somewhere else in the system. To cope with such events, we here extend our SFAM parametrization procedure $^{6}$ by the option to reparametrize locally, for which QM data from the QM-region calculation may be exploited. In general, the missing parameters must be obtained in an efficient way at a small fraction of the cost of the full-system parametrization.

At the start, we identify all parameters which are not covered by the existing set of SFAM parameters. To calculate the required reference data (i.e., optimized local geometries, Hessian matrix, atomic partial charges, bond orders), we fragment the whole system as explained in our original work on $\mathrm{SFAM}^{6}$ but perform calculations only on those fragments that were generated around the atoms involved in the bonds, angles, or dihedral angles with missing parameters. Subsequently, the parameters are optimized based on the calculated Hessians and local equilibrium geometries. Partial charges and connectivity information (obtained from Mayer ${ }^{104,105}$ covalent bond orders) are updated for all atoms and bonds for which new information is available (see our original work on $\mathrm{SFAM}^{6}$ for details).

Moreover, the dispersion coefficients are re-evaluated for the whole system due to the negligible additional computational effort associated with it. However, we note that the modification of these parameters upon changing the connectivity in the QM region may, in principle, introduce discontinuities in the energy gradients. As the bond breaking or formation process, in general, does not occur in close proximity to the $Q-\mathcal{E}$ boundary, it can be expected that this issue will hardly be a problem in practice but requires 
monitoring. Figure 2 provides an overview of the complete procedure.

Finally, we note that this strategy can be combined with a second approach toward flexible $\mathrm{QM}$ regions, i.e., with adaptive QM/MM schemes for molecular dynamics. ${ }^{106-110}$ These have been developed in recent years to allow for moving small molecules (e.g., solvent molecules) from the MM to the $\mathrm{QM}$ region (and vice versa) during an MD simulation while preserving a smooth description of the total energy.

2.7. Automated Selection of the QM Region. In this section, we introduce our algorithm for the selection of atoms for the QM region. Once a location of the QM region is provided, either based on structural characteristics that indicate chemical reactivity or by explicit manual determination, this location allows us to identify an atom around which the QM region is constructed (called "the center atom"). Our aim is to define an automated, universal, and data-driven procedure to find an accurate QM/MM model for the description of the reactive center when compared to a full QM calculation on the system or, if this is computationally not feasible, to the best possible estimate of that. First, one needs to define a descriptor to measure the accuracy of a given model. As mentioned in the Introduction, a previous study by Karelina and $\mathrm{Kulik}^{8}$ applied descriptors based on charge distribution. However, we focus on the forces on atoms in the proximity to the center atom because these relate directly to reasonable structures either in structure optimizations or molecular dynamics simulations.

For long-time molecular dynamics simulations (possibly with large basis sets), one cannot afford a QM region as large as, for instance, those in structure optimizations that require less than 100 single-point gradient calculations. Moreover, for small QM regions, it is also important to have a systematic approach toward a reliable solution. We therefore emphasize the importance of automation required to carry out a large number of exploratory calculations on candidate models of different sizes that need to be automatically set up, carried out, and then analyzed (including also the construction of the models) for the reliable and autonomous QM region determination to be applicable in a routine fashion.

In the following, we first explain how we construct QM regions around a given center atom automatically. Then, the selection criteria for the QM region are discussed. Finally, we elaborate on how to obtain reference data for systems where a full QM calculation is not feasible.

The construction of a QM region with a user-defined center atom represents a task analogous to the fragmentation step in our SFAM model generation. ${ }^{6}$ In the latter case, we construct one molecular fragment around each atom of the system under subsequent valence saturation with hydrogen atoms. This is achieved by first defining a sphere with radius $r_{0}$ around a selected atom and adding all atoms within it to the fragment. Second, all covalent bonds which were cut by the sphere's edge are identified and followed outward recursively until a covalent bond is reached at which the system can be divided and valence-saturated by a residue (currently, hydrogen-atom saturation has been implemented). Which bonds are considered cleavable is predetermined but can be adapted for a given system. For biochemical systems, $\mathrm{C}_{\mathrm{sp}^{3}}-\mathrm{X}$ bonds (with $\mathrm{X}=\mathrm{C}, \mathrm{N}$ ) can be considered a suitable choice because of their abundance in biological macromolecules. We emphasize that advanced embedding schemes ${ }^{45-49}$ such as frozen density embedding $^{47-62}$ may have the potential to replace this rulebased saturation approach. Combined with an initial radius between $r_{0}=5.5$ and $7.0 \AA$, our strategy resulted in a maximum fragment size of under 150 atoms for several of our example systems, ${ }^{6}$ which means that the required reference data can easily be calculated for these fragments with contemporary density functional theory.

To sample several model sizes and boundaries, we introduce a stochastic element to our automated QM/MM model construction. If a cleavable bond is reached, the system may be chosen to be split into QM and MM parts at that bond with some probability $p$. Naturally, the resulting set of QM regions contains duplicates because each QM region is constructed independently. Hence, the set of QM regions must be deduplicated. This straightforward approach is chosen over a systematic generation of all possible QM regions because of its simple implementation in the current fragmentation framework and the otherwise exploding number of possible QM regions of varying size, for which an exhaustive generation and selection process becomes unfeasible. Nevertheless, we point out that for small QM regions, the stochastic approach is also capable of generating all possible QM regions exhaustively up to a given size due to the efficiency of the fragmentation algorithm. Hence, the parameters $r_{0}$ and $p$ allow for adjusting the QM region size and the variation of sizes.

To filter and categorize the generated QM regions, we introduce two additional descriptors. The first one is the number of covalent bonds $m_{\text {link }}$ cut in the process of defining the QM region, which is equal to the number of link atoms in the resulting fragment. As a second feature of the generated QM regions, we introduce a symmetry measure $m_{\text {sym }}$

$$
m_{\text {sym }}=\frac{\bar{r}_{\mathrm{LDM}}}{\bar{r}_{\mathrm{MDQ}}}
$$

where $\bar{r}_{\mathrm{LDM}}$ is the mean distance of the central atom to the three least distant MM (LDM) atoms and $\bar{r}_{\mathrm{MDQ}}$ is the mean distance of the central atom to the three most distant QM (MDQ) atoms. This descriptor can be applied to assess the extent to which atoms are arranged aspherically around the reactive center.

To measure the reliability of some automatically produced QM/MM model $m$ in terms of how accurately the atomic forces in close proximity to the center atom are described compared to a reference ("ref"), we first select a set of $N_{\text {repr }}$ representative atoms that are closer than a cutoff of $r_{\text {repr }}$ to the center atom. The mean absolute error $\varepsilon_{k}{ }^{m}$ of the force components $\left(f_{x, m, k}, f_{y, m, k}\right.$, and $\left.f_{z, m, k}\right)$ for a given atom $k$ in this set is given by

$$
\begin{aligned}
\varepsilon_{k}^{m}= & \frac{1}{3}\left(\left|f_{x, \mathrm{ref}, k}-f_{x, m, k}\right|+\left|f_{y, \mathrm{ref}, k}-f_{y, m, k}\right|\right. \\
& \left.+\left|f_{z, \mathrm{ref}, k}-f_{z, m, k}\right|\right)
\end{aligned}
$$

The overall accuracy can then be measured by the mean of these errors

$$
\varepsilon_{\text {mean }}^{m}=\frac{1}{N_{\text {repr }}} \sum_{k=1}^{N_{\text {repr }}} \varepsilon_{k}^{m}
$$

The reference forces $\mathbf{f}_{\text {ref }, k}=\left(f_{x, \text { ref }, k}, f_{y, \text { ref }, k}, f_{z, \text { ref }, k}\right)^{\mathrm{T}}$ in eq 24 may be obtained from a QM calculation on a significantly larger system. As only one single-point gradient evaluation is necessary for this purpose, this is feasible for systems of several hundred atoms. Alternatively, we may obtain a reliable 


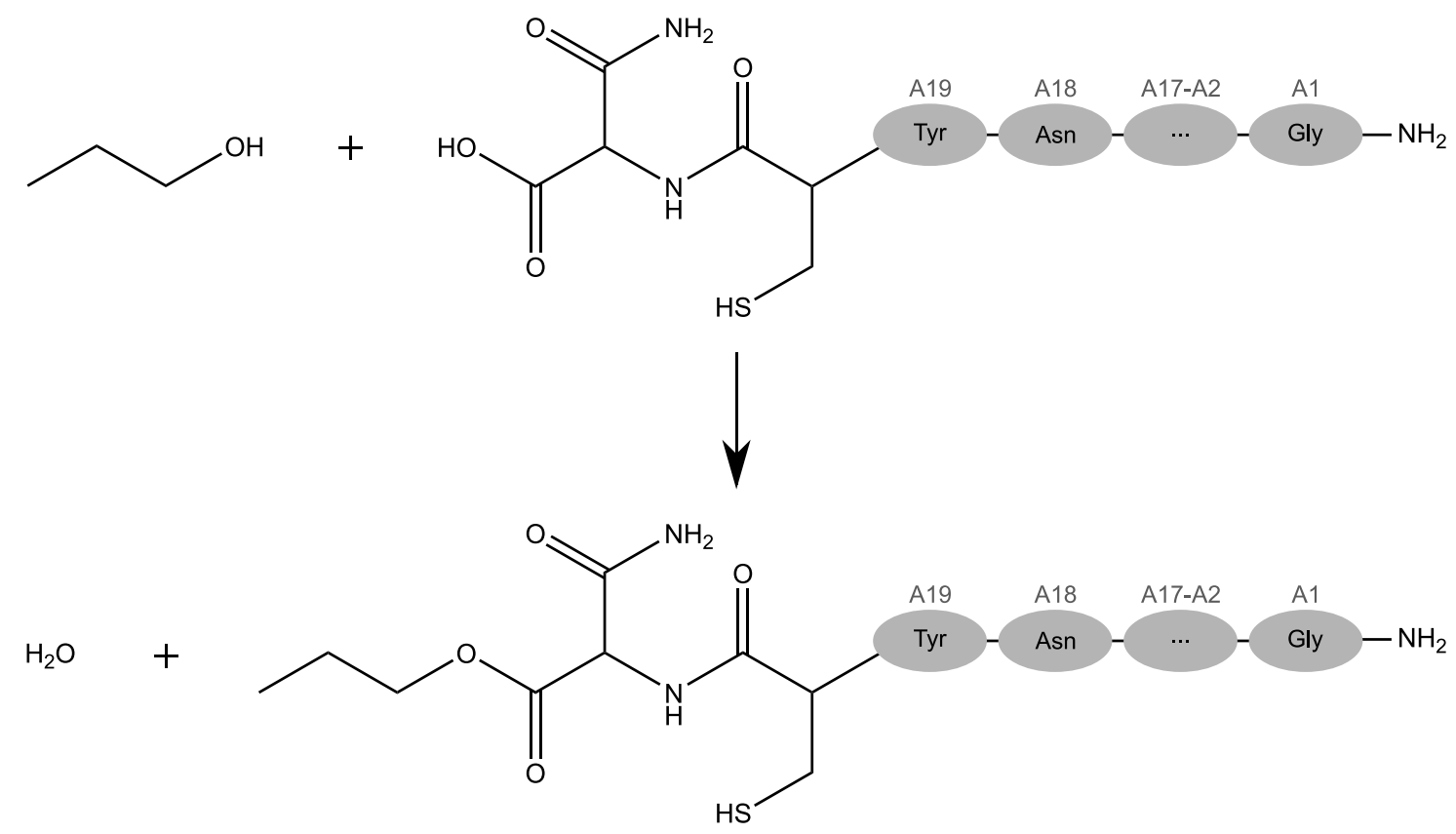

Figure 3. Reaction of 1-propanol with chain A of the peptide hormone insulin, which contains 21 amino acids, labeled A1-A21. The residues A20 (cysteine) and A21 (asparagine) are represented as Lewis structures. Note that this representation refrains from showing the disulfide bridge connecting two cysteine residues at positions A7 and A11.

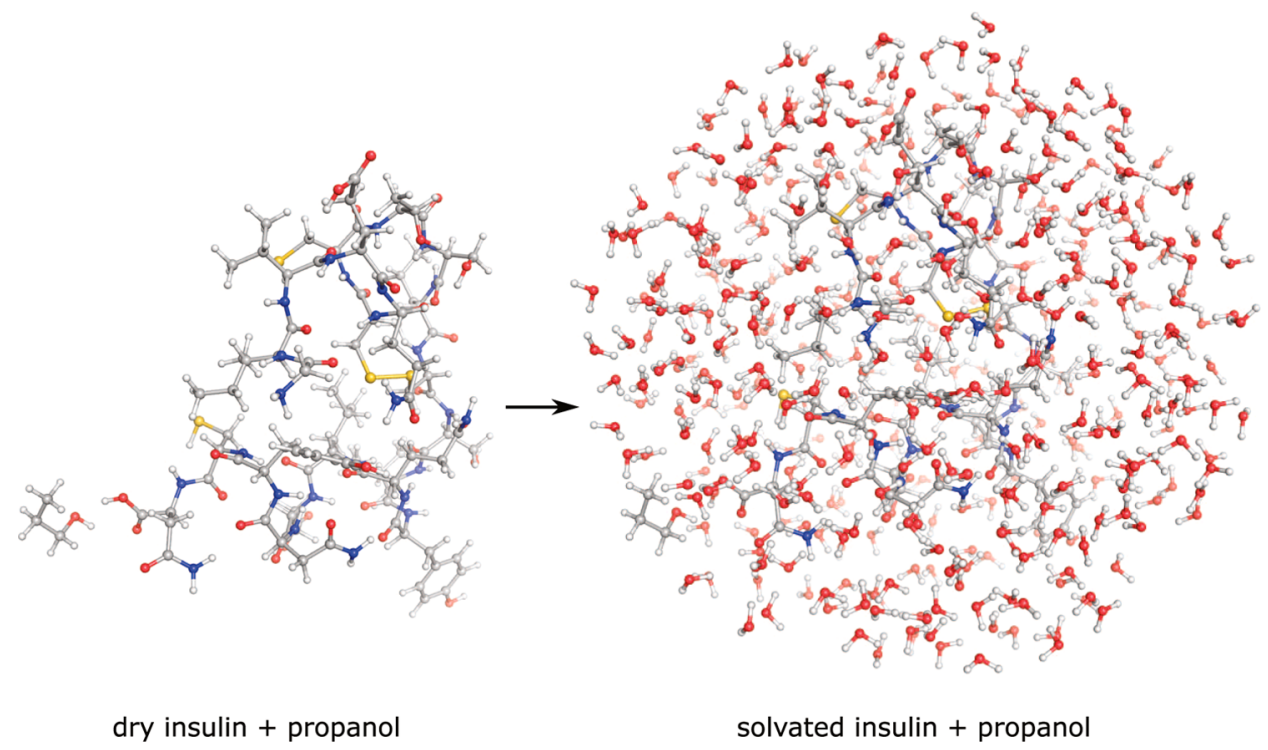

Figure 4. Dry (left) and microsolvated (right; $16 \AA$ water-molecule sphere) insulin with propanol as the reactant (cf. Figure 3) Atom coloring: carbon, gray; hydrogen, white; oxygen, red; nitrogen, blue; and sulfur, yellow.

estimate for the reference in the case of larger systems by averaging the force vectors obtained from a sample of $N_{\text {ref }}$ QM/SFAM models with large QM regions by choosing a radius $r_{0}$ that is as large as possible for a single-point calculation to be still feasible in a reasonable amount of time. It is important that this estimate is not based on a single reference model but on many different ones because it has been shown that one cannot be certain that molecular properties are converged even with $\mathrm{QM}$ regions of up to several hundred atoms. ${ }^{9-12}$ The comparison of several QM/ $\mathrm{MM}$ models with different QM/MM boundaries allows us to detect whether the atomic forces are converged with the QM region size that was chosen for the reference. If significant deviations exist between reference calculations, this can be detected and flagged by the algorithm automatically.

As a last remark, we note that one can deploy the domainbased local pair natural orbital coupled cluster methods ${ }^{11-113}$ as the QM part (allowing for large QM regions) to obtain accurate reaction barrier heights, ${ }^{87,114}$ which is crucial in mechanistic explorations for the subsequent kinetic analysis. ${ }^{115}$ Running QM/SFAM calculations with these QM methods is enabled through an interface to the quantum chemistry software ORCA. ${ }^{116,117}$

\section{RESULTS}

We demonstrate our automated QM/SFAM setup algorithm with two examples. For the first example, chain $\mathrm{A}$ of the 


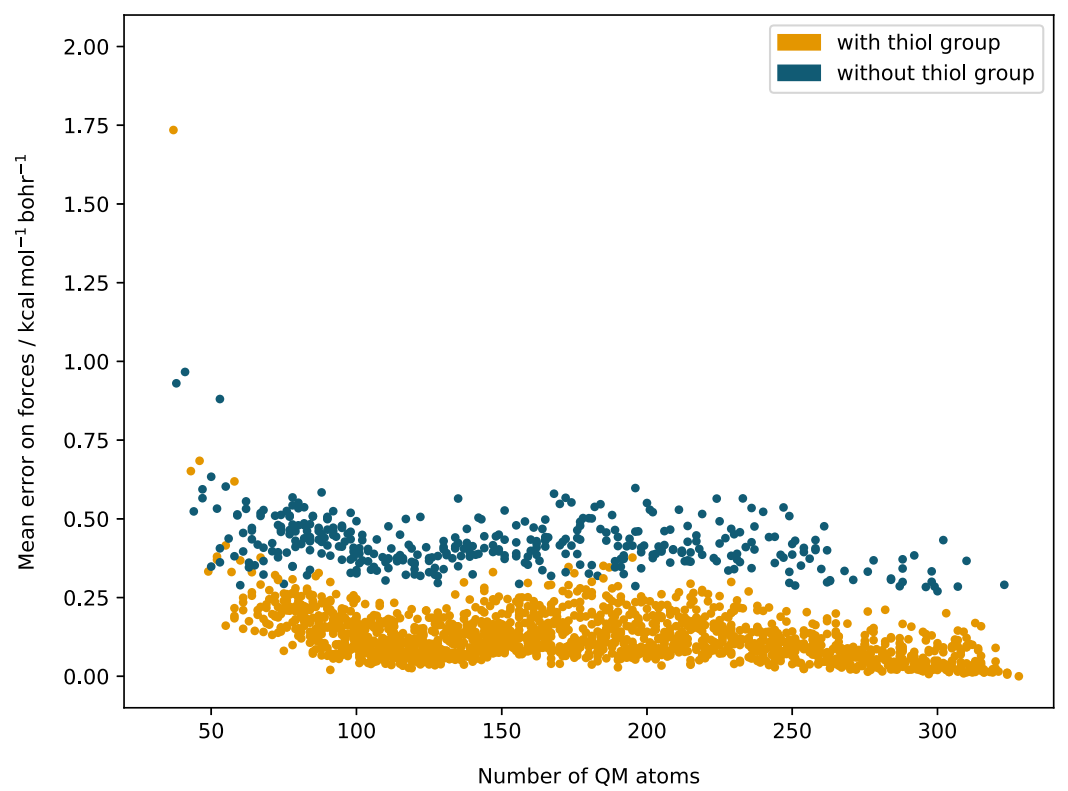

Figure 5. Mean error $\varepsilon_{\text {mean }}^{m}$ of atomic forces of all nonhydrogen atoms within $4.0 \AA$ of the central carbon atom in the generated QM/SFAM models for the unsolvated initial structure of insulin. Each point corresponds to a different QM/MM model $m$. The points are colored such that for the orange ones the thiol group $\mathrm{SH}_{5.7}$ is part of the QM region, whereas for the blue points, it is part of the environment. The point farthest to the right corresponds to the model representing the full $\mathrm{QM}$ calculation, which is taken as the reference for the error evaluation.

peptide hormone insulin ${ }^{118}$ was chosen because its size of slightly more than 300 atoms is large enough to test the effects of different QM regions on the QM/SFAM results while, at the same time, full QM calculations are still feasible so that a welldefined full QM reference is available. The initial structure was taken from the Protein Data Bank ${ }^{119}$ (PDB ID: 1AI0).

3.1. Construction of Structural Models. To study a chemical reaction in this system, we added a 1-propanol molecule in close proximity to the carboxylic acid group of the C-terminus of the chain (asparagine, A21), which serves as the initial structure of an esterification reaction. The product structure therefore contains a free water molecule and the propyl ester compound. This reaction is depicted in Figure 3. With the added alcohol as the reactant, the system consists of 328 atoms. We fully preoptimized the reactant and product structures with the PM3 semiempirical method ${ }^{120,121}$ applying the ORCA 4.2 quantum chemistry software. ${ }^{116,117}$ The subsequent DFT optimization with RI-PBE-D3(BJ)/def2$\mathrm{SVP}^{28,122-126}$ was limited to 10 optimization steps to obtain forces on all atoms that neither vanish nor acquire artificially large numerical values. The coordinates of these structures can be found in the Supporting Information.

For the reactant structure, a SFAM molecular mechanics model was parametrized in a fully automated fashion. ${ }^{6}$ The reference data, i.e., optimized structures, Hessians, Mayer bond orders, and atomic partial charges, were obtained for the RIPBE-D3(BJ)/def2-SVP electronic structure model with ORCA 4.2 driven by our software. ORCA Hirshfeld charges ${ }^{127}$ were converted to Charge Model 5 charges by our implementation of the published algorithm ${ }^{128}$ in our SCINE software. ${ }^{129}$

For the generation of the second example, we solvated the (dry) insulin peptide structure with water molecules. We applied the solvation tool of the ADF 2016.107 graphical user interface ${ }^{130}$ with which 418 water molecules were added (16 $\AA$ sphere, solute factor of 2.0 ), resulting in the second system that now comprises 1582 atoms in total. After the water molecules were added, the system was not relaxed. Again, we note that this is done deliberately to work with nonzero forces. The coordinates of the solvated structures can also be found in the Supporting Information (see Figure 4 for a ball-and-stick representation of both systems). The SFAM parametrization was carried out analogously to the unsolvated insulin system.

We note that for the parametrization, the bottleneck with respect to computing time is the reference-data generation step with all other tasks being completed in less than $5 \mathrm{~min}$ for the dry insulin peptide structure and less than $1 \mathrm{~h}$ for the solvated structure on a modern computing architecture with a single core. However, the time needed for the reference data generation can vary significantly depending on the number of cores chosen for parallel execution. In our setup, all reference calculations for the solvated structure were completed within a few days in 200 parallel calculations on four cores each.

For QM/SFAM calculations, the RI-PBE-D3(BJ)/def2$\mathrm{SVP}^{28,122-126}$ combination of density functional and basis set was applied for the QM region through an interface to the ORCA 4.2 software ${ }^{116,117}$ in both examples.

3.2. Dry Insulin. We first apply the QM-region generation algorithm of Section 2.7 to the structural model of dry insulin. The carbon atom of the carboxylic acid group of residue A21 (see Figure 3) was chosen as the center atom around which the QM region is constructed. We study whether the accuracy of the atomic forces close to the center of the QM region can be exploited to ensure reproducibility of the QM reference by the QM/SFAM model. For this purpose, a large variety of different QM regions was generated, including aspherical ones with a large value of $m_{\text {sym }}$ (see eq 23). We applied a small initial radius $r_{0}=5.5 \AA$ and a small cutting probability of $15 \%$ until $2000 \mathrm{QM} / \mathrm{SFAM}$ structural models were generated. The largest QM region obtained contained 328 atoms, which is identical to the whole system (full QM calculation), while the smallest QM region consists of only 32 atoms. More information about the distribution of QM region sizes and 


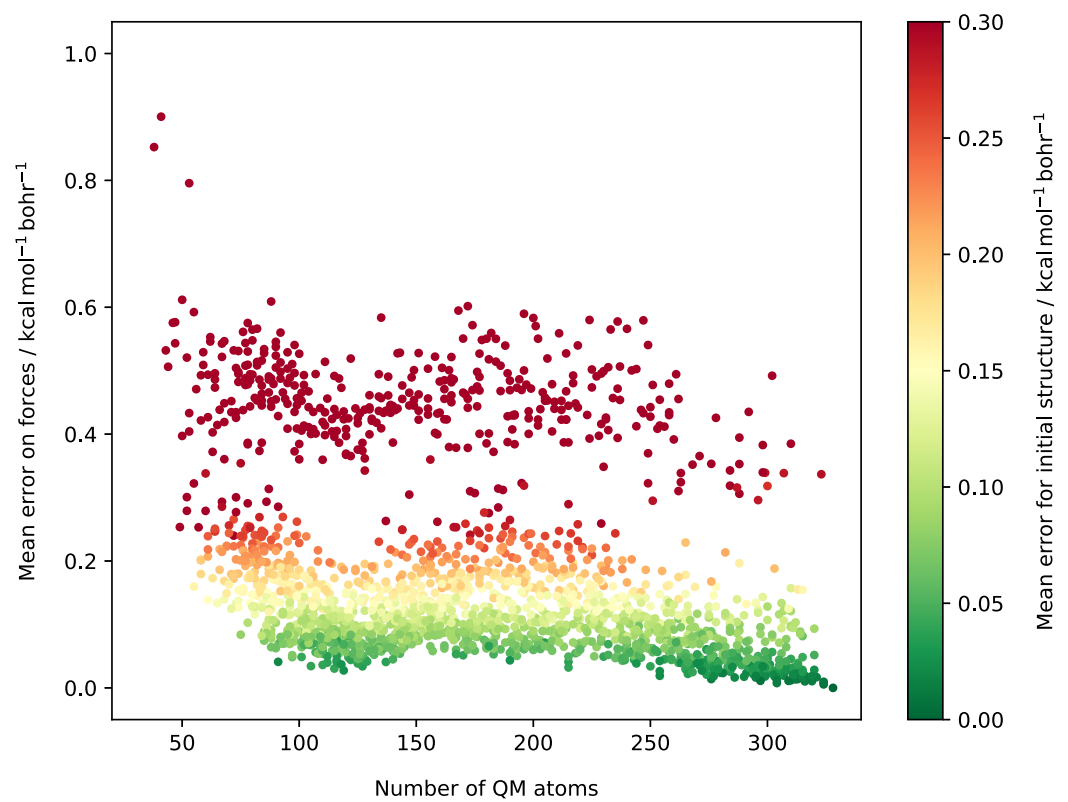

Figure 6. Mean error $\varepsilon_{\text {mean }}^{m}$ on atomic forces of all nonhydrogen atoms within $4.0 \AA$ of the central carbon atom for the generated QM/SFAM model for the unsolvated insulin final (product) structure. The model with the smallest QM region exhibits a significantly larger error than $1 \mathrm{kcal} \mathrm{mol}^{-1}$ $\mathrm{bohr}^{-1}$ (as in the case of the initial reactant structure; see Figure 5) and therefore has been omitted here. The colors encode the mean error that was obtained for the initial reactant structure with that model (i.e., the vertical axis in Figure 5). Models with errors larger than $0.3 \mathrm{kcal} \mathrm{mol}^{-1}$ $\mathrm{bohr}^{-1}$ on the initial structure are not further distinguished in color as these are not considered reasonable model candidates. This representation highlights that the accuracy found for the initial structure is matched by the accuracy obtained at a different location on the PES (here, the final product structure of the esterification reaction).

the distribution of symmetry scores $m_{\text {sym }}$ obtained by this algorithm can be found in the Supporting Information.

Figure 5 presents the results of evaluating the mean error $\varepsilon_{\text {mean }}^{m}$ on the atomic forces of all nonhydrogen atoms within $r_{\text {repr }}=4.0 \AA$ of the central carbon atom $\left(N_{\text {repr }}=10\right)$, according to eq 25 . We observe that only four models exhibit a large error of more than $0.75 \mathrm{kcal} \mathrm{mol}^{-1} \mathrm{bohr}^{-1}$. All of these models consist of less than $60 \mathrm{QM}$ atoms. Despite the obvious rationale that more $\mathrm{QM}$ atoms should result in higher accuracy, the mean error on the forces does not strictly decrease with the size of the QM region. This can be attributed to our choice to generate models with a large variance in asphericity (achieved by applying a small value for $p$ and measured by the symmetry score $m_{\text {sym }}$ ). Large QM regions with more than about $200 \mathrm{QM}$ atoms may be lacking a residue in close proximity to the center of the QM region, which would then result in a large error $\varepsilon_{\text {mean }}^{m}$. However, for the models with the smallest error for a given size of the QM region, the trend of a decreasing error is observed for models with more than $150 \mathrm{QM}$ atoms. QM regions that are very aspherical may be regarded as unsuitable and should not be considered in an application. This can be achieved by choosing a larger value for $p$, as well as by directly rejecting aspherical models with an improper value for $m_{\text {sym. }}$. We demonstrate this with our second example, the solvated insulin in Section 3.3, for which the expected trend of decreasing $\varepsilon_{\text {mean }}^{m}$ with increasing QM region size can be observed (see below).

Moreover, it can be easily seen in Figure 5 that the data split into two groups, which are separated by about $0.3 \mathrm{kcal} \mathrm{mol}^{-1}$ $\mathrm{bohr}^{-1}$. It follows from this observation that $\varepsilon_{\text {mean }}^{m}$ allows us to easily eliminate one of these groups from our consideration. The cause for this effect can be attributed to including or excluding the thiol group of the cysteine residue A20 from the QM atoms. The distance of the sulfur atom of this group to the central atom was $5.7 \AA$, which implies that it was not always included in the QM region. However, it was close enough to affect the esterification reaction significantly. We call this group $\mathrm{SH}_{5.7}$. The coloring in Figure 5 highlights this observation. It demonstrates that our descriptor $\varepsilon_{\text {mean }}^{m}$ is able to distinguish clearly the QM/MM models in which $\mathrm{SH}_{5.7}$ is part of the QM region from those where it is not. $98.4 \%$ of all models $m$ that contain $\mathrm{SH}_{5.7}$ in their QM region were able to reproduce the reference forces of the full QM calculation with a mean error $\varepsilon_{\text {mean }}^{m}$ of less than $0.3 \mathrm{kcal} \mathrm{mol}^{-1} \mathrm{bohr}^{-1}$, while all other models produced an error larger than $0.27 \mathrm{kcal} \mathrm{mol}^{-1} \mathrm{bohr}^{-1}$. With this example, in which the crucial functional group can be easily identified, we understand that it is possible to automatically and reliably sort out QM/SFAM models where an unreliable choice of $\mathrm{QM}$ region leads to large errors in atomic forces. Furthermore, we identified a second functional group (a carboxylic acid moiety $\left(\mathrm{COOH}_{9.5}\right)$ at a distance of 9.5 $\AA$ to the central carbon atom), for which an effect on the forces is observed. As this residue has a larger distance to the reaction center, its influence on the latter is smaller, which results in the observation that the corresponding groups of data are not well separated. Due to the small size of this effect, we refer to the Supporting Information for its visualization (differently colored version of Figure 5).

If a QM/SFAM model is able to accurately describe the forces in the reactant structures but poorly for intermediates, transition states, or products, it is not sensible to rely on this descriptor for the evaluation of the QM region selection protocol. Therefore, we evaluated the atomic forces on the same atoms as before for the final (product) structure of the reaction shown in Figure 3 by applying the same 2000 automatically selected QM/MM models to assess whether the models that led to a small error for the initial structure also performed well for the final structure. The results of this 


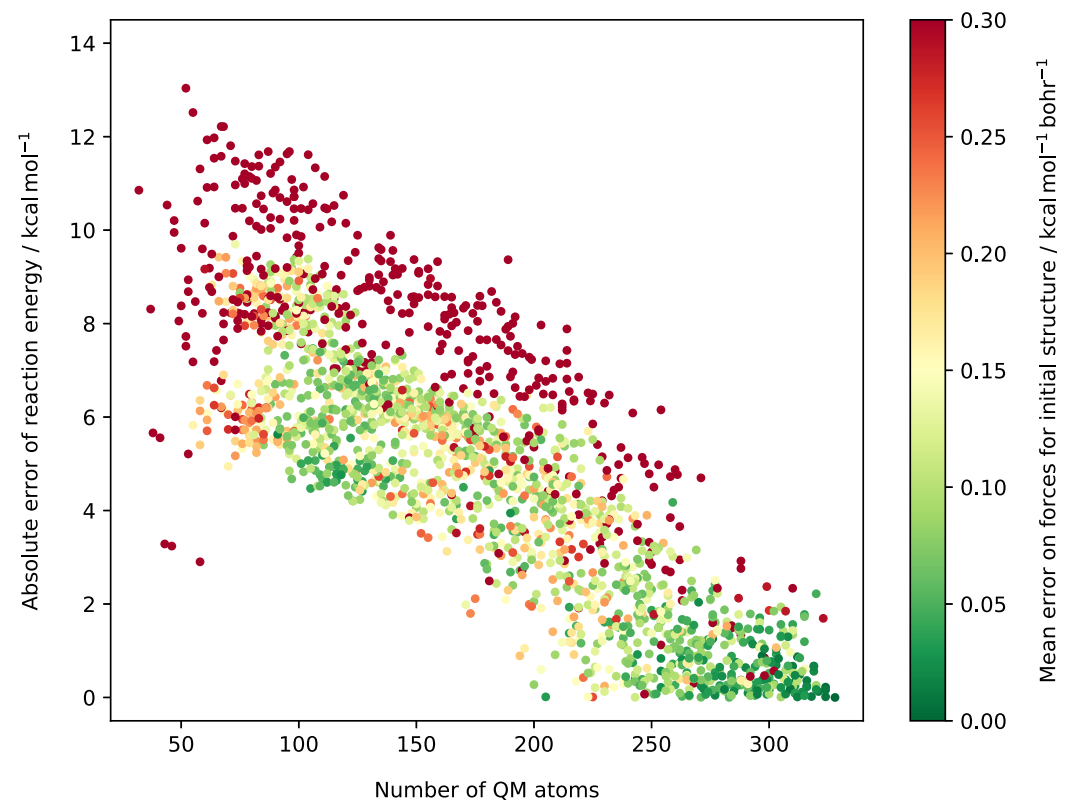

Figure 7. Absolute error of the esterification reaction energy for each of the generated QM/SFAM models of the unsolvated chain A of insulin. The colors correspond to the mean error on the atomic forces close to the reaction center obtained for the initial structure with that model (i.e., the vertical axis in Figure 5). Models with errors larger than $0.3 \mathrm{kcal} \mathrm{mol}^{-1} \mathrm{bohr}^{-1}$ on the initial structure are not further distinguished by color.

comparison are presented in Figure 6, in which we encode the accuracy of the models on the initial structure forces by their color. We observe an almost perfect agreement of force deviations measured in terms of $\varepsilon_{\text {mean }}^{m}$ for the initial structure and those for the final structure, indicating that our measure for the reliability of a selected QM region is likely to be transferable across a PES, at least for close-to-minimum energy structures.

Naturally, the size of the QM region may be different for other physical quantities and our assumption that the forces are most crucial for making a decision on the size, albeit reasonable from a structural point of view, needs to be scrutinized. Therefore, we now discuss whether those QM/ SFAM models that most accurately reproduced forces also deliver reliable energies. For the esterification reaction in dry insulin, the reaction energy calculated as the difference of reduced QM/SFAM energies (see Section 2.5, eq 21) obtained for the product and reactant structures is presented in Figure 7, where the same coloring scheme used for the forces of the product structure in Figure 6 is applied.

First, we observe that the average energy error decreases continuously with increasing QM region size. Second, the models that exhibited a large error on the forces (larger than $0.3 \mathrm{kcal} \mathrm{mol}^{-1} \mathrm{bohr}^{-1}$ ) are separated from the well-performing QM/SFAM models with only a small number of exceptions. This shows that models that were discarded after evaluating their accuracy on the forces are also expected to generate large errors in energy, confirming the reliability of our selection strategy. However, we also observe that for the well-performing models for which the reaction energy only fluctuates by less than $2 \mathrm{kcal} \mathrm{mol}^{-1}$ for a given QM region size, the accuracy of the forces does not map perfectly to the accuracy of the reaction energies (compared to Figure 6). Models with differences of less than $0.2 \mathrm{kcal} \mathrm{mol}^{-1} \mathrm{bohr}^{-1}$ on the forces are not distinguished in terms of the energies. However, we still reduce the error (compared to the most accurate QM/ SFAM model for a given QM region size) significantly by excluding the models with large errors on the forces; for instance, it is reduced from roughly 4 to $2 \mathrm{kcal} \mathrm{mol}^{-1}$ considering QM region sizes between 100 and 200 atoms.

Figure 7 shows that the model evaluation based on atomic forces can predict which models exhibit large errors of reaction energy. However, we observe that it cannot be guaranteed that the models with the smallest values of $\varepsilon_{\text {mean }}^{m}$ also exhibit the smallest energy errors. Considering these observations, we conclude that the descriptor $\varepsilon_{\text {mean }}^{m}$ can reliably eliminate choices of QM regions, which are lacking residues that significantly affect key physical quantities of the reaction. However, the fact that this descriptor is based on a single-point property (the atomic forces) results in both a crucial advantage and a drawback of the method. On the one hand, it allows us to efficiently test many model candidates in an automated fashion against reference data that are calculated with models with very large QM regions. On the other hand, we have shown that it cannot be guaranteed that the models with the smallest $\varepsilon_{\text {mean }}^{m}$ provide reaction energies that are within $1 \mathrm{kcal}$ $\mathrm{mol}^{-1}$ of the QM reference. Figure 7 demonstrates that in our example, this accuracy can only be achieved by applying very large QM regions with more than 250 atoms. During an exploration of a molecular reaction, we therefore stress the importance of applying models of several QM region sizes in single-point energy calculations to probe for convergence to closely monitor the uncertainties by which the QM region selections are affected. Respective algorithmic procedures can be included in automated workflows.

The energy errors discussed so far are, of course, given with respect to a DFT reference and therefore affected with some unknown uncertainty. We stress, however, that accurate quantum chemical methods, such as coupled cluster approaches ${ }^{87,114}$ with sufficiently high excitation rank and decent one-particle bases combined with basis-set exploration or explicit correlation factors, can be applied to obtain more accurate energies. Note that it may be beneficial to extend our QM region selection process by explicitly adding energy differences of two or more structures to our descriptor. 


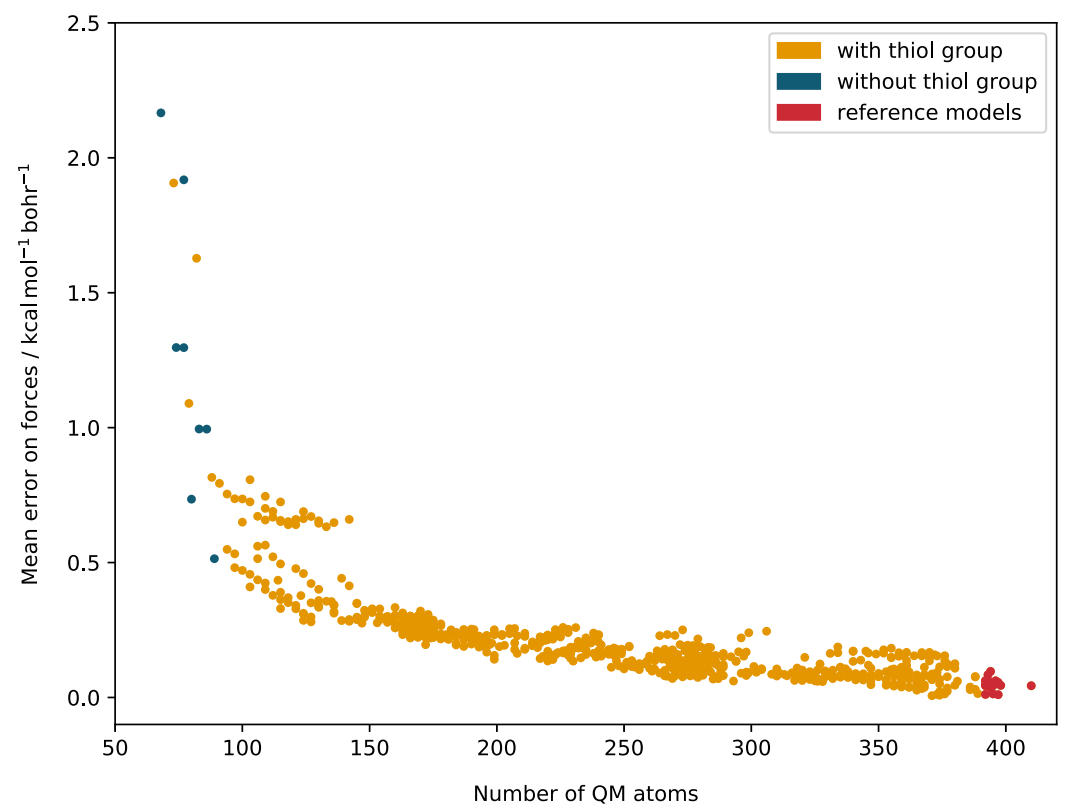

Figure 8. Mean error $\varepsilon_{\text {mean }}^{m}$ on the atomic forces of all nonhydrogen atoms within $4.0 \AA$ of the central carbon atom for the generated QM/SFAM models for the initial structure of solvated insulin. Each point corresponds to a different QM/SFAM model. The points are colored such that for the orange ones the thiol group $\mathrm{SH}_{5.7}$ is part of the $\mathrm{QM}$ region, while for the blue points, it is part of the environment. The red points correspond to the models for which the mean of the forces was taken as the reference (QM regions of at least 390 atoms). With the fragmentation settings applied here, which differ from those of unsolvated insulin, all models with more than $100 \mathrm{QM}$ atoms include the thiol group in the QM region.

Therefore, we designed our implementation in a modular fashion to allow for such extensions.

3.3. Solvated Insulin. With the example of solvated insulin, we demonstrate that it is possible to obtain reliable reference forces even for large systems for which one cannot routinely perform a full QM calculation on the whole system. As described in Section 3.1, the structures of the previous example after solvation with water are applied for this purpose.

For this solvated insulin structure, we do not want to generate a variety of different QM regions that is as large in number as the one in Section 3.2 because (i) this increases the total number of candidate models that needs to be tested (which is not practical in a routine QM/SFAM application) due to the larger total system size and (ii) the first example already demonstrated that very aspherical QM regions (e.g., large QM regions without the nearby $\mathrm{SH}_{5.7}$ group) do not provide accurate results. Hence, we set a larger probability of $p$ $=0.9$ for this example. The QM/SFAM models were generated by sampling $200 \mathrm{QM}$ regions for a given radius $r_{0}$ while increasing this value in steps of $0.1 \AA$ starting at $5.5 \AA$ and terminating at $11 \AA$, yielding 11000 models in total. After the deduplication process, 673 unique QM/SFAM models were created. The smallest QM region comprised 68 atoms, the largest 410 atoms. The obtained distribution of QM region sizes is provided in the Supporting Information.

As described in Section 2.7, we take the mean of $N_{\text {ref }}$ models with very large QM regions to obtain the reference forces. In this case, we assign all models with QM regions of more than 390 atoms to this set, resulting in $N_{\text {ref }}=18$. For the assessment of the models, the atomic forces of all nonhydrogen atoms within $4.0 \AA$ of the central carbon atom were considered $\left(N_{\text {repr }}=11\right)$. The analysis was performed on the initial (reactant) structure of the esterification reaction, and the results are presented in Figure 8.

Choosing a larger radius $r_{0}$ and cutting probability $p$ to generate the models with a larger $\mathrm{QM}$ region (in contrast to the setting in Section 3.2) resulted in the observation that all QM/SFAM models with more than $100 \mathrm{QM}$ atoms include the thiol group $\mathrm{SH}_{5.7}$ in the QM region. We also observed that generating the QM regions in a systematic way leads to the continuous increase of accuracy with increasing QM region size. The models taken as the reference (red points in Figure 8) do not show large fluctuations in accuracy. A mean deviation $\varepsilon_{\text {mean }}^{m}$ of $0.05 \mathrm{kcal} \mathrm{mol}^{-1} \mathrm{bohr}^{-1}$ for this set of models was obtained, and the maximum deviation $\varepsilon_{\max }^{m}$ was $0.1 \mathrm{kcal}$ $\mathrm{mol}^{-1} \mathrm{bohr}^{-1}$. From this, we can deduce that the functional groups that were present in some of the QM regions of these models, but not in all of them, do not have a significant effect on the forces. Hence, we can reliably apply the reference values obtained by this strategy. We stress that this approach is unavoidably limited by the maximum QM region size that is still computationally feasible. Therefore, sampling of several QM/SFAM models with QM regions of such size is crucial to obtain a reliable reference.

To select a QM region from these data, we consider the models with the smallest error on the forces for a given range of QM region sizes (constrained by the type of calculation and the available computational resources). To pick an example, we select a model with a QM region of $125 \pm 10$ atoms, which may be considered computationally feasible and of reasonable size for a variety of applications. Within the data presented in Figure 8 , this requirement is satisfied by 38 of the generated models. Note that one would typically generate only those models fulfilling the desired size requirement, and the number of candidates can be increased if deemed necessary and feasible (by varying $r_{0}$ and $p$ ).

We apply a tolerance of $0.05 \mathrm{kcal} \mathrm{mol}^{-1} \mathrm{bohr}^{-1}$ (based on the mean deviation in the reference models), which yields seven models with the highest accuracy of the forces as the remaining candidates. We stress that our approach is not able to discriminate between these models reliably, as the differences in performance of these models are smaller than 
the mean deviation in the reference models. An option to overcome this issue is to perform additional reference calculations on other structures on the PES and deploy these reference data to determine the optimal QM region. Calculating data for more than one point on the PES also facilitates the use of energy differences as a selection criterion.

A simpler alternative is to base the final selection on heuristic rules. We chose to apply the following: (i) models with fewer cuts at covalent bonds (i.e., a smaller number of link atoms $\left.m_{\text {link }}\right)$ are always preferred within this preselection and (ii) models with symmetry scores $m_{\text {sym }}$ (see eq 23) that are $50 \%$ larger than the minimal $m_{\text {sym }}$ are discarded to prevent an unphysical QM region to be selected due to error compensation. In our current implementation, we employ these rules to guide the final selection, which represents a systematic and reproducible process. However, we plan to extend this process in future work, as outlined above, based on additional reference data generation to obtain a final selection based purely on first-principles data.

Applying this selection strategy, a QM/SFAM model with a QM region of 127 atoms (excluding three link atoms) was selected in our example. The molecular structure of this QM region is depicted in Figure 9. The symmetry score of this QM region is $m_{\text {sym }}=1.24$, and the reaction energy error with this model was $1.96 \mathrm{kcal} \mathrm{mol}^{-1}$.

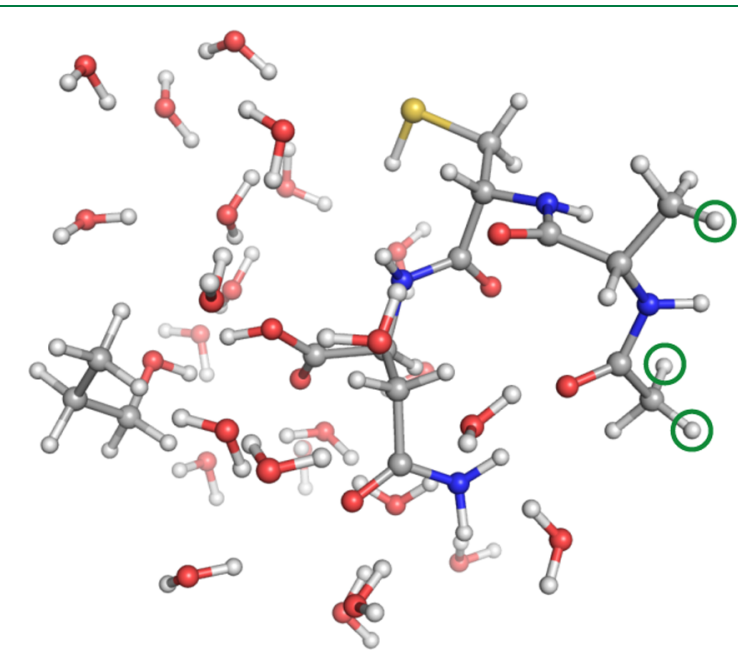

Figure 9. Molecular structure of the QM region of the automatically selected QM/SFAM model. As an example, we limited the acceptable size of this QM region to $125 \pm 10$ atoms. The green circles indicate the three hydrogen link atoms that are not present in the original structure but added for valence saturation of the QM region. Atom coloring: carbon, gray; hydrogen, white; oxygen, red; nitrogen, blue; and sulfur, yellow.

We emphasize that the construction of this QM/SFAM model and its selection over the other model candidates is fully automated in our implementation. This includes reference calculation management, which is automatized and parallelized in the same way as has been implemented for the SFAM parametrization process. We write the information about all of the necessary reference calculations into a MongoDB database, ${ }^{131}$ which is subsequently processed by $n$ instances of another program carrying out the calculations and storing the results back into the database. With this setup, $n$-fold parallelization of the data generation is enabled and therefore many QM/SFAM model candidates can be considered and tested efficiently. This is particularly important because most of the computing time needed by the quantum region selection algorithm can be attributed to the reference calculations. In our setup, each of the reference calculations (QM/SFAM models with very large quantum regions) was completed in less than $1 \mathrm{~h}$ applying eight cores per QM calculation.

Finally, it is pleasing to see that our automated protocol produces similarly sized QM regions like those that would be chosen manually by experienced $\mathrm{QM} / \mathrm{MM}$ practitioners. However, we stress that automatization is the key benefit here: (i) any human bias or prejudice on QM-region selection is avoided, (ii) full automatization no longer requires cumbersome human effort in $\mathrm{QM} / \mathrm{MM}$ model construction, and (iii) it also facilitates fully automated computational workflows. As a result, QM/MM calculations become easily accessible to nonexperts, and, by virtue of SFAM, they are likely to gain increased popularity for any sort of nanoscale atomistic simulation because of the first-principles character of all steps that do not impose any restriction on the application field (i.e., applications are not limited to biochemical macromolecules).

\section{CONCLUSIONS}

In this work, we reported a new QM/MM hybrid model for atomistic simulations which features a system-focused force field for minimized errors. We developed a fully automated setup of this QM/SFAM model, which by construction (i.e., by virtue of the salient features of SFAM) is not plagued with typical limitations of standard force fields (such as missing parameters for specific metal atoms in relevant valence states). However, if required, the methodology reported can be combined with such a standard force field [we implemented the general AMBER force field (GAFF) $\left.{ }^{132}\right]$. Our implementation will be made available within the open-source SCINE platform. ${ }^{129}$

As a result, the cumbersome manual setup of QM/MM models has been decisively alleviated up to the point where it can be driven in a fully automated way, which opens up new avenues for $\mathrm{QM} / \mathrm{MM}$ approaches; e.g., (i) in interactive approaches, ${ }^{101,102}$ where operator-defined abrupt changes of focus occur, (ii) in situations of quickly changing reactive sites because of highly mobile or volatile reactants, or (iii) in studies of complex chemical systems with varying environments such as enzymes generated by high-throughput-directed evolution.

If, during a molecular exploration, new covalent bonds are formed and then shifted to the MM region (e.g., because the QM region is moved to a different local region of the full structure), molecular mechanics parameters may be missing for this new chemical environment in the classical region. However, the SFAM ansatz allows our implementation to quickly reparametrize this new local situation with only minimal computational effort. Furthermore, our implementation is flexible enough to allow for two or more (unconnected) $\mathrm{QM}$ regions in the model.

Our automated model construction process also allows for the generation and application of several models with differently sized QM regions in parallel. This enables us to estimate and control the uncertainty of the model constantly, even in fully automated exploration setups. ${ }^{99,100}$ As was demonstrated in Section 3.2, this is of great importance when calculating physical quantities (e.g., reaction energies) that are not directly related to the atomic forces on which we based our model selection criterion. However, the modular nature of our 
implementation allows for extending the selection criteria to include additional quantities if necessary.

\section{ASSOCIATED CONTENT}

\section{SI Supporting Information}

The Supporting Information is available free of charge at https://pubs.acs.org/doi/10.1021/acs.jctc.1c00178.

Additional data characterizing the generated QM regions; the documentation of the slight effect of the carboxylic acid moiety for the selection of the optimal $\mathrm{QM}$ region of the dry insulin system (PDF)

Cartesian coordinates of dry and solvated insulin (ZIP)

\section{AUTHOR INFORMATION}

\section{Corresponding Author}

Markus Reiher - Laboratorium für Physikalische Chemie, ETH Zürich, 8093 Zürich, Switzerland; ㅇorcid.org/00000002-9508-1565; Email: markus.reiher@

phys.chem.ethz.ch

\section{Author}

Christoph Brunken - Laboratorium für Physikalische Chemie, ETH Zürich, 8093 Zürich, Switzerland

Complete contact information is available at: https://pubs.acs.org/10.1021/acs.jctc.1c00178

\section{Notes}

The authors declare no competing financial interest.

\section{ACKNOWLEDGMENTS}

C.B. gratefully acknowledges support by a Kekulé Ph.D. fellowship of the Fonds der Chemischen Industrie. The authors thank the Schweizerischer Nationalfonds for generous support (Projects 200021_182400 to M.R. and 200021_172950-1 (C.B.) to PD Dr. Thomas Hofstetter).

\section{REFERENCES}

(1) Senn, H. M.; Thiel, W. QM/MM Methods for Biological Systems. Top. Curr. Chem. 2007, 268, 173-290.

(2) Groenhof, G. Biomolecular Simulations: Methods in Molecular Biology; Humana Press: Totowa, NJ, 2013; pp 43-66.

(3) Riniker, S. Fixed-charge atomistic force fields for molecular dynamics simulations in the condensed phase: An overview. J. Chem. Inf. Model. 2018, 58, 565-578.

(4) Warshel, A.; Levitt, M. Theoretical studies of enzymic reactions: Dielectric, electrostatic and steric stabilization of the carbonium ion in the reaction of lysozyme. J. Mol. Biol. 1976, 103, 227-249.

(5) Thiel, W.; Hummer, G. Nobel 2013 Chemistry: Methods for computational chemistry. Nature 2013, 504, No. 96.

(6) Brunken, C.; Reiher, M. Self-Parametrizing System-Focused Atomistic Models. J. Chem. Theory Comput. 2020, 16, 1646-1665.

(7) Ramakrishnan, R.; Dral, P. O.; Rupp, M.; von Lilienfeld, O. A. Big data meets quantum chemistry approximations: The $\Delta$-machine learning approach. J. Chem. Theory Comput. 2015, 11, 2087-2096.

(8) Karelina, M.; Kulik, H. J. Systematic quantum mechanical region determination in QM/MM simulation. J. Chem. Theory Comput. 2017, 13, 563-576.

(9) Sumowski, C. V.; Ochsenfeld, C. A convergence study of QM/ $\mathrm{MM}$ isomerization energies with the selected size of the QM region for peptidic systems. J. Phys. Chem. A 2009, 113, 11734-11741.

(10) Flaig, D.; Beer, M.; Ochsenfeld, C. Convergence of electronic structure with the size of the QM region: example of QM/MM NMR shieldings. J. Chem. Theory Comput. 2012, 8, 2260-2271.
(11) Roßbach, S.; Ochsenfeld, C. Influence of Coupling and Embedding Schemes on QM Size Convergence in QM/MM Approaches for the Example of a Proton Transfer in DNA. J. Chem. Theory Comput. 2017, 13, 1102-1107.

(12) Kulik, H. J.; Zhang, J.; Klinman, J. P.; Martinez, T. J. How large should the $\mathrm{QM}$ region be in $\mathrm{QM} / \mathrm{MM}$ calculations? The case of catechol O-methyltransferase. J. Phys. Chem. B 2016, 120, 1138111394.

(13) Hu, L.; Söderhjelm, P.; Ryde, U. On the convergence of QM/ MM energies. J. Chem. Theory Comput. 2011, 7, 761-777.

(14) Liao, R.-Z.; Thiel, W. Convergence in the QM-only and QM/ MM modeling of enzymatic reactions: A case study for acetylene hydratase. J. Comput. Chem. 2013, 34, 2389-2397.

(15) Retegan, M.; Neese, F.; Pantazis, D. A. Convergence of QM/ MM and cluster models for the spectroscopic properties of the oxygen-evolving complex in photosystem II. J. Chem. Theory Comput. 2013, 9, 3832-3842.

(16) Grimme, S. A General Quantum Mechanically Derived Force Field (QMDFF) for Molecules and Condensed Phase Simulations. J. Chem. Theory Comput. 2014, 10, 4497-4514.

(17) Vanduyfhuys, L.; Vandenbrande, S.; Verstraelen, T.; Schmid, R.; Waroquier, M.; Van Speybroeck, V. QuickFF: A program for a quick and easy derivation of force fields for metal-organic frameworks from ab initio input. J. Comput. Chem. 2015, 36, 1015-1027.

(18) Wang, R.; Ozhgibesov, M.; Hirao, H. Partial hessian fitting for determining force constant parameters in molecular mechanics. J. Comput. Chem. 2016, 37, 2349-2359.

(19) Wang, R.; Ozhgibesov, M.; Hirao, H. Analytical hessian fitting schemes for efficient determination of force-constant parameters in molecular mechanics. J. Comput. Chem. 2018, 39, 307-318.

(20) Li, Z.; Kermode, J. R.; De Vita, A. Molecular dynamics with onthe-fly machine learning of quantum-mechanical forces. Phys. Rev. Lett. 2015, 114, No. 096405.

(21) Chmiela, S.; Tkatchenko, A.; Sauceda, H. E.; Poltavsky, I.; Schütt, K. T.; Müller, K.-R. Machine learning of accurate energyconserving molecular force fields. Sci. Adv. 2017, 3, No. e1603015.

(22) Glielmo, A.; Sollich, P.; De Vita, A. Accurate interatomic force fields via machine learning with covariant kernels. Phys. Rev. B 2017, 95, No. 214302.

(23) Chmiela, S.; Sauceda, H. E.; Müller, K.-R.; Tkatchenko, A. Towards exact molecular dynamics simulations with machine-learned force fields. Nat. Commun. 2018, 9, No. 3887.

(24) Amabilino, S.; Bratholm, L. A.; Bennie, S. J.; O’Connor, M. B.; Glowacki, D. R. Training Atomic Neural Networks Using FragmentBased Data Generated in Virtual Reality. 2020, arXiv:physics/ 2007.02824. arXiv.org e-Print archive. http://arxiv.org/abs/2007. 02824 .

(25) Zhang, Y.-J.; Khorshidi, A.; Kastlunger, G.; Peterson, A. A. The potential for machine learning in hybrid $\mathrm{QM} / \mathrm{MM}$ calculations. J. Chem. Phys. 2018, 148, No. 241740.

(26) Böselt, L.; Thürlemann, M.; Riniker, S. Machine Learning in QM/MM Molecular Dynamics Simulations of Condensed-Phase Systems. 2020, arXiv:physics/2010.11610. arXiv.org e-Print archive. http://arxiv.org/abs/2010.11610.

(27) Johnson, E. R.; Mackie, I. D.; DiLabio, G. A. Dispersion interactions in density-functional theory. J. Phys. Org. Chem. 2009, 22, $1127-1135$

(28) Grimme, S.; Antony, J.; Ehrlich, S.; Krieg, H. A consistent and accurate $\mathrm{ab}$ initio parametrization of density functional dispersion correction (DFT-D) for the 94 elements H-Pu. J. Chem. Phys. 2010, 132, No. 154104.

(29) Grimme, S.; Ehrlich, S.; Goerigk, L. Effect of the damping function in dispersion corrected density functional theory. J. Comput. Chem. 2011, 32, 1456-1465.

(30) Grimme, S. Density functional theory with London dispersion corrections. Wiley Interdiscip. Rev.: Comput. Mol. Sci. 2011, 1, 211228. 
(31) Field, M. J.; Bash, P. A.; Karplus, M. A combined quantum mechanical and molecular mechanical potential for molecular dynamics simulations. J. Comput. Chem. 1990, 11, 700-733.

(32) Singh, U. C.; Kollman, P. A. A combined ab initio quantum mechanical and molecular mechanical method for carrying out simulations on complex molecular systems: Applications to the $\mathrm{CH} 3 \mathrm{Cl}+\mathrm{Cl}-$ exchange reaction and gas phase protonation of polyethers. J. Comput. Chem. 1986, 7, 718-730.

(33) Maseras, F.; Morokuma, K. IMOMM: A new integrated ab initio+ molecular mechanics geometry optimization scheme of equilibrium structures and transition states. J. Comput. Chem. 1995, $16,1170-1179$

(34) Eichler, U.; Kölmel, C. M.; Sauer, J. Combining ab initio techniques with analytical potential functions for structure predictions of large systems: Method and application to crystalline silica polymorphs. J. Comput. Chem. 1997, 18, 463-477.

(35) Antes, I.; Thiel, W. Combined Quantum Mechanical and Molecular Mechanical Methods; American Chemical Society: Washington, DC, 1998; Chapter 4, pp 50-65.

(36) Das, D.; Eurenius, K. P.; Billings, E. M.; Sherwood, P.; Chatfield, D. C.; Hodošček, M.; Brooks, B. R. Optimization of quantum mechanical molecular mechanical partitioning schemes: Gaussian delocalization of molecular mechanical charges and the double link atom method. J. Chem. Phys. 2002, 117, 10534-10547.

(37) Swart, M. AddRemove: A new link model for use in QM/MM studies. Int. J. Quantum Chem. 2003, 91, 177-183.

(38) Théry, V.; Rinaldi, D.; Rivail, J.-L.; Maigret, B.; Ferenczy, G. G. Quantum mechanical computations on very large molecular systems: The local self-consistent field method. J. Comput. Chem. 1994, 15, 269-282.

(39) Monard, G.; Loos, M.; Théry, V.; Baka, K.; Rivail, J.-L. Hybrid classical quantum force field for modeling very large molecules. Int. J. Quantum Chem. 1996, 58, 153-159.

(40) Assfeld, X.; Rivail, J.-L. Quantum chemical computations on parts of large molecules: the ab initio local self consistent field method. Chem. Phys. Lett. 1996, 263, 100-106.

(41) Ferré, N.; Assfeld, X.; Rivail, J.-L. Specific force field parameters determination for the hybrid ab initio QM/MM LSCF method. J. Comput. Chem. 2002, 23, 610-624.

(42) Gao, J.; Amara, P.; Alhambra, C.; Field, M. J. A generalized hybrid orbital (GHO) method for the treatment of boundary atoms in combined QM/MM calculations. J. Phys. Chem. A 1998, 102, 47144721.

(43) Amara, P.; Field, M. J.; Alhambra, C.; Gao, J. The generalized hybrid orbital method for combined quantum mechanical/molecular mechanical calculations: formulation and tests of the analytical derivatives. Theor. Chem. Acc. 2000, 104, 336-343.

(44) Garcia-Viloca, M.; Gao, J. Generalized hybrid orbital for the treatment of boundary atoms in combined quantum mechanical and molecular mechanical calculations using the semiempirical parameterized model 3 method. Theor. Chem. Acc. 2004, 111, 280-286.

(45) Huzinaga, S.; Cantu, A. Theory of separability of many-electron systems. J. Chem. Phys. 1971, 55, 5543-5549.

(46) Manby, F. R.; Stella, M.; Goodpaster, J. D.; Miller, T. F. A Simple, Exact Density-Functional-Theory Embedding Scheme. J. Chem. Theory Comput. 2012, 8, 2564-2568.

(47) Fornace, M. E.; Lee, J.; Miyamoto, K.; Manby, F. R.; Miller, T. F., III Embedded mean-field theory. J. Chem. Theory Comput. 2015, $11,568-580$.

(48) Hégely, B.; Nagy, P. R.; Ferenczy, G. G.; Kállay, M. Exact density functional and wave function embedding schemes based on orbital localization. J. Chem. Phys. 2016, 145, No. 064107.

(49) Lee, S. J.; Welborn, M.; Manby, F. R.; Miller, T. F., III Projection-Based Wavefunction-in-DFT Embedding. Acc. Chem. Res. 2019, 52, 1359-1368.

(50) Wesolowski, T. A.; Warshel, A. Frozen density functional approach for ab initio calculations of solvated molecules. J. Phys. Chem. A 1993, 97, 8050-8053.
(51) Neugebauer, J.; Louwerse, M. J.; Baerends, E. J.; Wesolowski, T. A. The merits of the frozen-density embedding scheme to model solvatochromic shifts. J. Chem. Phys. 2005, 122, No. 094115.

(52) Neugebauer, J.; Jacob, C. R.; Wesolowski, T. A.; Baerends, E. J. An Explicit Quantum Chemical Method for Modeling Large Solvation Shells Applied to Aminocoumarin C151. J. Phys. Chem. A 2005, 109, $7805-7814$.

(53) Neugebauer, J.; Louwerse, M. J.; Belanzoni, P.; Wesolowski, T. A.; Baerends, E. J. Modeling solvent effects on electron-spinresonance hyperfine couplings by frozen-density embedding. J. Chem. Phys. 2005, 123, No. 114101.

(54) Neugebauer, J.; Baerends, E. J. Exploring the Ability of FrozenDensity Embedding to Model Induced Circular Dichroism. J. Phys. Chem. A 2006, 110, 8786-8796.

(55) Jacob, C. R.; Neugebauer, J.; Jensen, L.; Visscher, L. Comparison of frozen-density embedding and discrete reaction field solvent models for molecular properties. Phys. Chem. Chem. Phys. 2006, 8, 2349-2359.

(56) Wesołowski, T. A. Embedding a multideterminantal wave function in an orbital-free environment. Phys. Rev. A 2008, 77, No. 012504.

(57) Jacob, C. R.; Neugebauer, J.; Visscher, L. A flexible implementation of frozen-density embedding for use in multilevel simulations. J. Comput. Chem. 2008, 29, 1011-1018.

(58) Pernal, K.; Wesolowski, T. A. Orbital-free effective embedding potential: Density-matrix functional theory case. Int. J. Quantum Chem. 2009, 109, 2520-2525.

(59) Fux, S.; Jacob, C. R.; Neugebauer, J.; Visscher, L.; Reiher, M. Accurate frozen-density embedding potentials as a first step towards a subsystem description of covalent bonds. J. Chem. Phys. 2010, 132, No. 164101.

(60) Jacob, C. R.; Neugebauer, J. Subsystem density-functional theory. Wiley Interdiscip. Rev.: Comput. Mol. Sci. 2014, 4, 325-362.

(61) Wesolowski, T. A.; Shedge, S.; Zhou, X. Frozen-density embedding strategy for multilevel simulations of electronic structure. Chem. Rev. 2015, 115, 5891-5928.

(62) Mühlbach, A. H.; Reiher, M. Quantum system partitioning at the single-particle level. J. Chem. Phys. 2018, 149, No. 184104.

(63) Huheey, J. E.; Keiter, E. A.; Keiter, R. L.; Medhi, O. K. Inorganic Chemistry: Principles of Structure and Reactivity; Pearson Education India, 2006.

(64) Greenwood, N. N.; Earnshaw, A. Chemistry of the Elements; Elsevier, 2012.

(65) Walker, R. C.; Crowley, M. F.; Case, D. A. The implementation of a fast and accurate QM/MM potential method in Amber. J. Comput. Chem. 2008, 29, 1019-1031.

(66) Eurenius, K. P.; Chatfield, D. C.; Brooks, B. R.; Hodoscek, M. Enzyme mechanisms with hybrid quantum and molecular mechanical potentials. I. Theoretical considerations. Int. J. Quantum Chem. 1996, $60,1189-1200$.

(67) Ryde, U. The coordination of the catalytic zinc ion in alcohol dehydrogenase studied by combined quantum-chemical and molecular mechanics calculations. J. Comput.-Aided Mol. Des. 1996, 10, $153-164$.

(68) Sherwood, P.; de Vries, A. H.; Collins, S. J.; Greatbanks, S. P.; Burton, N. A.; Vincent, M. A.; Hillier, I. H. Computer simulation of zeolite structure and reactivity using embedded cluster methods. Faraday Discuss. 1997, 106, 79-92.

(69) De Vries, A. H.; Sherwood, P.; Collins, S. J.; Rigby, A. M.; Rigutto, M.; Kramer, G. J. Zeolite structure and reactivity by combined quantum-chemical- classical calculations. J. Phys. Chem. B 1999, 103, 6133-6141.

(70) Sherwood, P.; de Vries, A. H.; Guest, M. F.; Schreckenbach, G.; Catlow, C. A.; French, S. A.; Sokol, A. A.; Bromley, S. T.; Thiel, W.; Turner, A. J.; Billeter, S.; Terstegen, F.; Thiel, S.; Kendrick, J.; Rogers, S. C.; Casci, J.; Watson, M.; King, F.; Karlsen, E.; Sjøvoll, M.; Fahmi, A.; Schäfer, A.; Lennartz, C. QUASI: A general purpose implementation of the $\mathrm{QM} / \mathrm{MM}$ approach and its application to problems in catalysis. J. Mol. Struct.: THEOCHEM 2003, 632, 1-28. 
(71) König, P. H.; Hoffmann, M.; Frauenheim, T.; Cui, Q. A critical evaluation of different $\mathrm{QM} / \mathrm{MM}$ frontier treatments with SCC-DFTB as the QM method. J. Phys. Chem. B 2005, 109, 9082-9095.

(72) Lin, H.; Truhlar, D. G. Redistributed charge and dipole schemes for combined quantum mechanical and molecular mechanical calculations. J. Phys. Chem. A 2005, 109, 3991-4004.

(73) Eichinger, M.; Tavan, P.; Hutter, J.; Parrinello, M. A hybrid method for solutes in complex solvents: Density functional theory combined with empirical force fields. J. Chem. Phys. 1999, 110, 10452-10467.

(74) Amara, P.; Field, M. J. Evaluation of an ab initio quantum mechanical/molecular mechanical hybrid-potential link-atom method. Theor. Chem. Acc. 2003, 109, 43-52.

(75) Hall, R. J.; Hindle, S. A.; Burton, N. A.; Hillier, I. H. Aspects of hybrid $\mathrm{QM} / \mathrm{MM}$ calculations: the treatment of the $\mathrm{QM} / \mathrm{MM}$ interface region and geometry optimization with an application to chorismate mutase. J. Comput. Chem. 2000, 21, 1433-1441.

(76) Vreven, T.; Morokuma, K.; Farkas, Ö.; Schlegel, H. B.; Frisch, M. J. Geometry optimization with QM/MM, ONIOM, and other combined methods. I. Microiterations and constraints. J. Comput. Chem. 2003, 24, 760-769.

(77) Vreven, T.; Morokuma, K. Hybrid Methods: ONIOM(QM:MM) and QM/MM. Annu. Rep. Comput. Chem. 2006, 2, 3551.

(78) Kästner, J.; Thiel, S.; Senn, H. M.; Sherwood, P.; Thiel, W. Exploiting $\mathrm{QM} / \mathrm{MM}$ capabilities in geometry optimization: A microiterative approach using electrostatic embedding. J. Chem. Theory Comput. 2007, 3, 1064-1072.

(79) Melaccio, F.; Olivucci, M.; Lindh, R.; Ferré, N. Unique QM/ MM potential energy surface exploration using microiterations. Int. J. Quantum Chem. 2011, 111, 3339-3346.

(80) Metz, S.; Kästner, J.; Sokol, A. A.; Keal, T. W.; Sherwood, P. Chem Shell-a modular software package for $\mathrm{QM} / \mathrm{MM}$ simulations. Wiley Interdiscip. Rev.: Comput. Mol. Sci. 2014, 4, 101-110.

(81) Caprasecca, S.; Jurinovich, S.; Viani, L.; Curutchet, C.; Mennucci, B. Geometry optimization in polarizable QM/MM models: the induced dipole formulation. J. Chem. Theory Comput. 2014, 10, No. 1588.

(82) Bosia, F.; Brunken, C.; Grimmel, S. A.; Haag, M. P.; Heuer, M. A.; Simm, G. N.; Sobez, J.-G.; Steiner, M.; Unsleber, J. P.; Vaucher, A. C.; Weymuth, T.; Reiher, M. SCINE Utilities, release 2.0.0, 2020. https://doi.org/10.5281/zenodo.3828692.

(83) Bryan, J. G.; Wadsworth, G. P. Introduction to Probability and Random Variables; McGraw-Hill: New York, 1960.

(84) Feller, W. An Introduction to Probability Theory and Its Applications; John Wiley \& Sons, 2008; Vol. 2.

(85) Weymuth, T.; Proppe, J.; Reiher, M. Statistical Analysis of Semiclassical Dispersion Corrections. J. Chem. Theory Comput. 2018, 14, 2480-2494.

(86) Proppe, J.; Gugler, S.; Reiher, M. Gaussian Process-Based Refinement of Dispersion Corrections. J. Chem. Theory Comput. 2019, $15,6046-6060$.

(87) Claeyssens, F.; Harvey, J. N.; Manby, F. R.; Mata, R. A.; Mulholland, A. J.; Ranaghan, K. E.; Schütz, M.; Thiel, S.; Thiel, W.; Werner, H.-J. High-Accuracy Computation of Reaction Barriers in Enzymes. Angew. Chem., Int. Ed. 2006, 45, 6856-6859.

(88) Walker, R. C.; Mercer, I. P.; Gould, I. R.; Klug, D. R. Comparison of basis set effects and the performance of $\mathrm{ab}$ initio and DFT methods for probing equilibrium fluctuations. J. Comput. Chem. 2007, 28, 478-490.

(89) Martí, M. A.; Capece, L.; Bikiel, D. E.; Falcone, B.; Estrin, D. A. Oxygen affinity controlled by dynamical distal conformations: the soybean leghemoglobin and the Paramecium caudatum hemoglobin cases. Proteins 2007, 68, 480-487.

(90) Mata, R. A.; Werner, H.-J.; Thiel, S.; Thiel, W. Toward accurate barriers for enzymatic reactions: $\mathrm{QM} / \mathrm{MM}$ case study on $\mathrm{p}$ hydroxybenzoate hydroxylase. J. Chem. Phys. 2008, 128, No. 01B610.
(91) Liao, R.-Z.; Thiel, W. Comparison of QM-only and QM/MM models for the mechanism of tungsten-dependent acetylene hydratase. J. Chem. Theory Comput. 2012, 8, 3793-3803.

(92) Polyak, I.; Reetz, M. T.; Thiel, W. Quantum mechanical/ molecular mechanical study on the mechanism of the enzymatic Baeyer-Villiger reaction. J. Am. Chem. Soc. 2012, 134, 2732-2741.

(93) Berraud-Pache, R.; Navizet, I. QM/MM calculations on a newly synthesised oxyluciferin substrate: new insights into the conformational effect. Phys. Chem. Chem. Phys. 2016, 18, 27460-27467.

(94) Bathelt, C. M.; Zurek, J.; Mulholland, A. J.; Harvey, J. N. Electronic structure of compound I in human isoforms of cytochrome P450 from QM/MM modeling. J. Am. Chem. Soc. 2005, 127, 1290012908.

(95) Geronimo, I.; Paneth, P. A DFT and ONIOM study of C-H hydroxylation catalyzed by nitrobenzene 1, 2-dioxygenase. Phys. Chem. Chem. Phys. 2014, 16, 13889-13899.

(96) Cooper, A. M.; Kästner, J. Averaging Techniques for Reaction Barriers in QM/MM Simulations. ChemPhysChem 2014, 15, 32643269.

(97) Finkelmann, A. R.; Senn, H. M.; Reiher, M. Hydrogenactivation mechanism of $[\mathrm{Fe}]$ hydrogenase revealed by multi-scale modeling. Chem. Sci. 2014, 5, 4474-4482.

(98) Ke, Z.; Abe, S.; Ueno, T.; Morokuma, K. Catalytic Mechanism in Artificial Metalloenzyme: QM/MM Study of Phenylacetylene Polymerization by Rhodium Complex Encapsulated in apo-Ferritin. J. Am. Chem. Soc. 2012, 134, 15418-15429.

(99) Simm, G. N.; Vaucher, A. C.; Reiher, M. Exploration of Reaction Pathways and Chemical Transformation Networks. J. Phys. Chem. A 2019, 123, 385-399.

(100) Unsleber, J. P.; Reiher, M. The Exploration of Chemical Reaction Networks. Annu. Rev. Phys. Chem. 2020, 71, 121-142.

(101) Haag, M. P.; Vaucher, A. C.; Bosson, M.; Redon, S.; Reiher, M. Interactive chemical reactivity exploration. ChemPhysChem 2014, $15,3301-3319$.

(102) Vaucher, A. C.; Haag, M. P.; Reiher, M. Real-time feedback from iterative electronic structure calculations. J. Comput. Chem. 2016, $37,805-812$.

(103) Grimme, S. Semiempirical GGA-type density functional constructed with a long-range dispersion correction. J. Comput. Chem. 2006, 27, 1787-1799.

(104) Mayer, I. Charge bond order and valence in the AB initio SCF theory. Chem. Phys. Lett. 1983, 97, 270-274.

(105) Mayer, I. On bond orders and valences in the ab initio quantum chemical theory. Int. J. Quantum Chem. 1986, 29, 73-84.

(106) Heyden, A.; Lin, H.; Truhlar, D. G. Adaptive partitioning in combined quantum mechanical and molecular mechanical calculations of potential energy functions for multiscale simulations. J. Phys. Chem. B 2007, 111, 2231-2241.

(107) Bulo, R. E.; Ensing, B.; Sikkema, J.; Visscher, L. Toward a practical method for adaptive QM/MM simulations. J. Chem. Theory Comput. 2009, 5, 2212-2221.

(108) Mones, L.; Jones, A.; Götz, A. W.; Laino, T.; Walker, R. C.; Leimkuhler, B.; Csányi, G.; Bernstein, N. The adaptive buffered force $\mathrm{QM} / \mathrm{MM}$ method in the CP2K and AMBER software packages. J. Comput. Chem. 2015, 36, 633-648.

(109) Zheng, M.; Waller, M. P. Adaptive quantum mechanics/ molecular mechanics methods. Wiley Interdiscip. Rev.: Comput. Mol. Sci. 2016, 6, 369-385.

(110) Duster, A. W.; Wang, C.-H.; Garza, C. M.; Miller, D. E.; Lin, $\mathrm{H}$. Adaptive quantum/molecular mechanics: What have we learned, where are we, and where do we go from here? Wiley Interdiscip. Rev.: Comput. Mol. Sci. 2017, 7, No. e1310.

(111) Neese, F.; Wennmohs, F.; Hansen, A. Efficient and accurate local approximations to coupled-electron pair approaches: An attempt to revive the pair natural orbital method. J. Chem. Phys. 2009, 130, No. 114108.

(112) Neese, F.; Hansen, A.; Liakos, D. G. Efficient and accurate approximations to the local coupled cluster singles doubles method 
using a truncated pair natural orbital basis. J. Chem. Phys. 2009, 131, No. 064103.

(113) Hansen, A.; Liakos, D. G.; Neese, F. Efficient and accurate local single reference correlation methods for high-spin open-shell molecules using pair natural orbitals. J. Chem. Phys. 2011, 135, No. 214102.

(114) Bistoni, G.; Polyak, I.; Sparta, M.; Thiel, W.; Neese, F. Toward accurate $\mathrm{QM} / \mathrm{MM}$ reaction barriers with large $\mathrm{QM}$ regions using domain based pair natural orbital coupled cluster theory. J. Chem. Theory Comput. 2018, 14, 3524-3531.

(115) Proppe, J.; Reiher, M. Mechanism deduction from noisy chemical reaction networks. J. Chem. Theory Comput. 2018, 15, 357370.

(116) Neese, F. The ORCA program system. Wiley Interdiscip. Rev.: Comput. Mol. Sci. 2012, 2, 73-78.

(117) Neese, F. Software update: the ORCA program system, version 4.0. Wiley Interdiscip. Rev.: Comput. Mol. Sci. 2018, 8, No. e1327.

(118) Sonksen, P.; Sonksen, J. Insulin: understanding its action in health and disease. Br. J. Anaesth. 2000, 85, 69-79.

(119) Bernstein, F. C.; Koetzle, T. F.; Williams, G. J.; Meyer, E. F., Jr; Brice, M. D.; Rodgers, J. R.; Kennard, O.; Shimanouchi, T.; Tasumi, M. The Protein Data Bank: a computer-based archival file for macromolecular structures. J. Mol. Biol. 1977, 112, 535-542.

(120) Stewart, J. J. Optimization of parameters for semiempirical methods I. Method. J. Comput. Chem. 1989, 10, 209-220.

(121) Stewart, J. J. Optimization of parameters for semiempirical methods II. Applications. J. Comput. Chem. 1989, 10, 221-264.

(122) Whitten, J. L. Coulombic potential energy integrals and approximations. J. Chem. Phys. 1973, 58, 4496-4501.

(123) Dunlap, B. I.; Connolly, J.; Sabin, J. On some approximations in applications of X $\alpha$ theory. J. Chem. Phys. 1979, 71, 3396-3402.

(124) Vahtras, O.; Almlöf, J.; Feyereisen, M. Integral approximations for LCAO-SCF calculations. Chem. Phys. Lett. 1993, 213, 514-518.

(125) Perdew, J. P.; Burke, K.; Ernzerhof, M. Generalized Gradient Approximation Made Simple. Phys. Rev. Lett. 1996, 77, 3865-3868.

(126) Weigend, F.; Ahlrichs, R. Balanced basis sets of split valence, triple zeta valence and quadruple zeta valence quality for $\mathrm{H}$ to $\mathrm{Rn}$ : Design and assessment of accuracy. Phys. Chem. Chem. Phys. 2005, 7, 3297-3305.

(127) Hirshfeld, F. L. Bonded-atom fragments for describing molecular charge densities. Theor. Chim. Acta 1977, 44, 129-138.

(128) Marenich, A. V.; Jerome, S. V.; Cramer, C. J.; Truhlar, D. G. Charge Model 5: An Extension of Hirshfeld Population Analysis for the Accurate Description of Molecular Interactions in Gaseous and Condensed Phases. J. Chem. Theory Comput. 2012, 8, 527-541.

(129) SCINE-Software for Chemical Interaction Networks. http://scine.ethz.ch/ (accessed July 7, 2020).

(130) te Velde, G.; Bickelhaupt, F. M.; Baerends, E. J.; Fonseca Guerra, C.; van Gisbergen, S. J. A.; Snijders, J. G.; Ziegler, T. Chemistry with ADF. J. Comput. Chem. 2001, 22, 931-967.

(131) MongoDB 3.2; MongoDB Inc., 2020. www.mongodb.com.

(132) Wang, J.; Wolf, R. M.; Caldwell, J. W.; Kollman, P. A.; Case, D. A. Development and testing of a general amber force field. J. Comput. Chem. 2004, 25, 1157-1174. 